\title{
A preliminary world checklist of fern-mining
}

\section{insects}

\author{
Jie Yang ${ }^{\ddagger}$, Xuexiong Wang ${ }^{\ddagger}$, Kevin Jan Duffy§, Xiaohua Dai ${ }^{\ddagger}, \mid$ \\ $\ddagger$ Leafminer Group, School of Life Sciences, Gannan Normal University, Ganzhou, China \\ $\S$ Institute of Systems Science, Durban University of Technology, Durban, South Africa \\ | National Navel-Orange Engineering Research Center, Ganzhou, China
}

Corresponding author: Xiaohua Dai (ecoinformatics@gmail.com)

Academic editor: Torsten Dikow

Received: 06 Jan 2021 | Accepted: 15 Mar 2021 | Published: 25 Mar 2021

Citation: Yang J, Wang X, Duffy KJ, Dai X (2021) A preliminary world checklist of fern-mining insects.

Biodiversity Data Journal 9: e62839. https://doi.org/10.3897/BDJ.9.e62839

\begin{abstract}
Compared to the leaf-miners and stem-miners on flowering plants, the miners on ferns (including both Lycopodiophyta and Polypodiophyta in the broad sense) are less known. Knowledge of miners and their host plants is essential to fully understand plant-insect interactions. Although there are many scattered records on fern miners, a worldwide checklist has not been reported.

We provide a preliminary checklist of fern-mining insects and their host plants worldwide. Altogether, we found records for 128 species and 18 families of fern miners, mainly that feed on Dennstaedtiaceae, Equisetaceae, Polypodiaceae and Aspleniaceae. Fern miners belonged to four orders: Diptera (51 species; 39.8\%), Coleoptera (33 species; 25.8\%), Lepidoptera (28 species; $21.9 \%$ ) and Hymenoptera (16 species; $12.5 \%$ ). They are primarily known from the Palaearctic Region, Nearctic Region, Neotropical Region and Oriental Region.
\end{abstract}

\section{Keywords}

fern, leaf miner, host plant, plant-insect interactions, plant apparency hypothesis 


\section{Introduction}

Leaf/stem miners are endophagous insects whose larvae feed on parenchyma or epidermal cells and form visually distinctive feeding tunnels, i.e. 'mines' on the leaves or stems (Dai et al. 2018, Eiseman 2020b, Liu et al. 2015, Sinclair and Hughes 2010). The mines can provide useful hints on insect species identities, insect life histories, insect behaviour and insect-plant interactions (Dai et al. 2018). Fewer miner groups (e.g. gracillariid moths, agromyzid flies and leaf-mining chrysomelids) can utilise over 100 plant families (Dai et al. 2017, De Prins and De Prins 2020, Santiago-Blay 2004, Spencer 1990), which are mainly angiosperm families, such as Fagaceae and Myrtaceae (Dai et al. 2018).

Ferns (Pteridophyta, including both Lycopodiophyta and Polypodiophyta in the broad sense) are the second largest group of vascular plants, just after angiosperms (Dai et al. 2020, Schneider et al. 2004). With lower nutrition, higher defensive chemicals and no flowers, the interspecific associations between ferns and insects are often overlooked (Mehltreter et al. 2010, Weintraub et al. 1995). However, ferns used to be the primary food resource for insects before the thriving of angiosperms (Mehltreter et al. 2010). In fact, fossil records indicate that ferns and insects have co-evolved for at least 300 myr (Chandra and Srivastava 2003). Some ferns have nectaries and domatia, which could attract ants to be bodyguards (Mehltreter et al. 2010). Moreover, some insects mimic the soral crypsis of ferns to escape from their natural enemies (Barker et al. 2005, Patra et al. 2008). Some researchers hypothesise that fern-feeding insects should have fewer species, genera and families than those of seed plants (Weintraub et al. 1995), while others suggest that the richness of fern-feeding insects is largely underestimated (Auerbach and Hendrix 1980, Mehltreter et al. 2010). The possible underestimation might be deduced from the following facts: (1) many fewer investigations have been performed for wild ferns than for cultivated ferns or invasive ferns (Fountain-Jones et al. 2012); (2) many more fern herbivores have been discovered in the comprehensive screening of bio-control agents for pest ferns (Mehltreter et al. 2010); (3) no noticeable difference has been found between leaf herbivory loss of ferns and that of seed plants (Chandra and Srivastava 2003); and (4) the possible biases of plant apparency (i.e. body size, distribution range and individual numbers (Dai et al. 2017)) are not considered for phytophagous insects on ferns in comparison to those on seed plants (Auerbach and Hendrix 1980).

Fern-feeding insects could be classified as generalists and specialists. Most fern-feeding generalists tend to be classified as leaf-chewing or sap-sucking, while most specialists are classified as leaf-mining, gall-forming or spore-feeding (Mehltreter et al. 2010). By far, the miners on ferns are much less known than those on seed plants; although there are scattered records on publications and websites (De Prins and De Prins 2020, Edmunds et al. 2020, Ellis 2020, Eiseman 2020b, Pitkin et al. 2019, Santiago-Blay 2004, Spencer 1990), few comprehensive reviews on fern miners have been provided and a worldwide checklist has not been reported. In this study, we will compile a preliminary checklist of fern-mining insects and their host plants throughout the world, which could provide meaningful information to the study of plant-insect-environment interactions. 


\section{Material and methods}

The names and hosts of fern miners were obtained from websites, books and articles. Most publications were retrieved from the Web of Science (https:// www.webofknowledge.com) and Google Scholar (https://scholar.google.com), while the others were obtained from reference lists of the websites and retrieved publications. According to the Taxonomic Name Resolution Service (http://tnrs.iplantcollaborative.org/ TNRSapp.html), the host fern's scientific names were verified and corrected. The number of species in a fern family was obtained from the Catalogue of Life (http:// www.catalogueoflife.org/). Based on two recent mega-trees (Smith and Brown 2018, Zanne et al. 2014), 'GBOTB.extended.tre' is the latest and largest dated phylogenetic tree for vascular plants, with 74533 species, 10587 genera and all extant 479 families (Jin and Qian 2019). The R package 'V. PhyloMaker' (Jin and Qian 2019) can bind undetermined plant taxa to the backbone phylogeny of 'GBOTB.extended.tre' and generates the customised tree we needed (Dai et al. 2020). Here, we obtained the phylogenetic tree of our host fern families using the above method. Bivariate linear regression was fitted with Past 4.04 (Hammer et al. 2001).

Both leaf-miners and stem-borers have been found in the same insect family (e.g. Buprestidae, Cossidae and Blasticotomidae) or even in the same genus (e.g. Amauromyza, Melanagromyza, Phytomyza and Zygoneura) (Eiseman 2020b, Hering 1951, Shcherbakov 2006, Woodley and Janzen 1995). Occasionally, the same species could change their feeding habits from leaf-mining to stem-mining or stem-boring, when the younger larvae transform into the older larvae, when the leaf is too small to offer enough food or when leaves and stems do not differ significantly (Eiseman 2020b, Hering 1951). Such phenomena can be found in Heliozela hammoniella (= Heliozela betulae) (Heliozelidae), Marmara spp. (Gracillariidae), Ophiomyia spp. (Agromyzidae), Phyllocnistis spp. (Gracillariidae), Scaptomyza graminum (= Scaptomyzella incana) (Drosophilidae), Zygoneura calthella (Sciaridae), Temnosira czurhini (Pallopteridae) and many other species (Eiseman 2020b, Ellis 2020, Hering 1951, Kato 2002). There are transitions amongst leaf-mining, stem-mining, leaf-boring and stem boring (Hering 1951). Moreover, most ferns are herbaceous, with developed parenchyma in the stems (Crang et al. 2018). Therefore, we incorporated fern borers into fern miners for this article (Suppl. material 1). Some suspected insect species are not included in this study (e.g. Correia et al. 2020, Santiago-Blay 2004).

The miners' biogeographical regions followed Juan J. Morrone's system (Morrone 2002). For detailed information about fern miners associated with each host plant species, the original sources should be consulted. 


\section{Results}

We recorded 128 species and 18 families of fern miners (Table 1; Suppl. material 2), including Agromyzidae, Anthomyiidae, Drosophilidae, Chironomidae, Pallopteridae, Buprestidae, Chrysomelidae, Curculionidae, Crambidae, Noctuidae, Tineidae, Tortricidae, Cosmopterigidae, Gelechiidae, Hepialidae, Psychidae, Blasticotomidae and Tenthredinidae. They were primarily distributed in the Palaearctic Region, Nearctic Region and Oriental Region of the Northern Hemisphere and the Neotropical Region between the Tropic of Cancer and the Tropic of Capricorn (Table 1). One explanation for this distribution pattern could be that the land area in the Northern Hemisphere is almost double that of the Southern Hemisphere. Another reason might be that the investigations on leaf-mining insects and their host plants are more thorough in the Northern than in the Southern Hemisphere (Sinclair and Hughes 2008, Sinclair and Hughes 2008, Sinclair and Hughes 2010).

\section{Table 1.}

A preliminary world checklist of fern miners and their biological information.

\begin{tabular}{|c|c|c|c|c|}
\hline Miner family & Miner species & Host fern & $\begin{array}{l}\text { Biogeographical } \\
\text { regions }\end{array}$ & References \\
\hline \multicolumn{5}{|l|}{ Diptera } \\
\hline Agromyzidae & $\begin{array}{l}\text { Chromatomyia } \\
\text { cheilanthus Garg }{ }^{\star 1}\end{array}$ & $\begin{array}{l}\text { Cheilanthes } \\
\text { virga-aure }\end{array}$ & Oriental Region & Spencer 1990 \\
\hline Agromyzidae & $\begin{array}{l}\text { Chromatomyia dorsata } \\
\text { Hendel }^{2}\end{array}$ & $\begin{array}{l}\text { Asplenium } \\
\text { ceterach }\end{array}$ & Palaearctic Region & Ellis 2020, Spencer 1990 \\
\hline Agromyzidae & $\begin{array}{l}\text { Chromatomyia } \\
\text { dryoptericola } \\
\text { Sasakawa }^{3}\end{array}$ & Dryopteris lacera & Palaearctic Region & \multirow[t]{3}{*}{$\begin{array}{l}\text { Sasakawa 2010, Spencer } \\
1990\end{array}$} \\
\hline Agromyzidae & $\begin{array}{l}\text { Chromatomyia } \\
\text { dryoptericola } \\
\text { Sasakawa }\end{array}$ & $\begin{array}{l}\text { Asplenium } \\
\text { pinnatifidum }\end{array}$ & Palaearctic Region & \\
\hline Agromyzidae & $\begin{array}{l}\text { Chromatomyia } \\
\text { dryoptericola } \\
\text { Sasakawa }\end{array}$ & $\begin{array}{l}\text { Lepisorus } \\
\text { thunbergianus }\end{array}$ & Palaearctic Region & \\
\hline Agromyzidae & $\begin{array}{l}\text { Chromatomyia } \\
\text { masumiae Sasakawa }\end{array}$ & $\begin{array}{l}\text { Lepisorus } \\
\text { thunbergianus }\end{array}$ & Palaearctic Region & Sasakawa 2010 \\
\hline Agromyzidae & $\begin{array}{l}\text { Chromatomyia } \\
\text { scolopendri Robineau } \\
\text { Desvoidy }^{4}\end{array}$ & $\begin{array}{l}\text { Asplenium ruta- } \\
\text { muraria }\end{array}$ & Palaearctic Region & \multirow{3}{*}{$\begin{array}{l}\text { Civelek 2002, Dempewolf } \\
2001 \text {, Ellis 2020, } \\
\text { Sasakawa 2010, Spencer } \\
1990\end{array}$} \\
\hline Agromyzidae & $\begin{array}{l}\text { Chromatomyia } \\
\text { scolopendri Robineau } \\
\text { Desvoidy }\end{array}$ & $\begin{array}{l}\text { Asplenium } \\
\text { scolopendrium }\end{array}$ & Palaearctic Region & \\
\hline Agromyzidae & $\begin{array}{l}\text { Chromatomyia } \\
\text { scolopendri Robineau } \\
\text { Desvoidy }\end{array}$ & $\begin{array}{l}\text { Asplenium } \\
\text { septentrionale }\end{array}$ & Palaearctic Region & \\
\hline
\end{tabular}




\begin{tabular}{|c|c|c|c|c|}
\hline Miner family & Miner species & Host fern & $\begin{array}{l}\text { Biogeographical } \\
\text { regions }\end{array}$ & References \\
\hline Agromyzidae & $\begin{array}{l}\text { Chromatomyia } \\
\text { scolopendri Robineau } \\
\text { Desvoidy }\end{array}$ & $\begin{array}{l}\text { Polypodium } \\
\text { vulgare }\end{array}$ & Palaearctic Region & \\
\hline Agromyzidae & $\begin{array}{l}\text { Liriomyza equiseti de } \\
\text { Meijere }^{5}\end{array}$ & $\begin{array}{l}\text { Equisetum } \\
\text { arvense }\end{array}$ & $\begin{array}{l}\text { Nearctic and } \\
\text { Palaearctic Regions }\end{array}$ & $\begin{array}{l}\text { Eiseman 2020b, Ellis } \\
\text { 2020, George 2014, } \\
\text { Lonsdale 2017, Spencer } \\
1990\end{array}$ \\
\hline Agromyzidae & $\begin{array}{l}\text { Liriomyza occipitalis } \\
\text { Hendel }^{6}\end{array}$ & $\begin{array}{l}\text { Equisetum } \\
\text { arvense }\end{array}$ & Palaearctic Region & $\begin{array}{l}\text { Ellis } 2020, \text { George } 2014 \text {, } \\
\text { Spencer } 1990\end{array}$ \\
\hline Agromyzidae & $\begin{array}{l}\text { Liriomyza nordica } \\
\text { Spencer* }\end{array}$ & Equisetum sp. & Nearctic Region & Eiseman 2020b \\
\hline Agromyzidae & $\begin{array}{l}\text { Liriomyza virgo } \\
\text { Zetterstedt }^{7}\end{array}$ & $\begin{array}{l}\text { Equisetum } \\
\text { fluviatile }\end{array}$ & $\begin{array}{l}\text { Nearctic and } \\
\text { Palaearctic Regions }\end{array}$ & \multirow{2}{*}{$\begin{array}{l}\text { Eiseman 2020b, Ellis } \\
\text { 2020, George 2014, } \\
\text { Lonsdale 2017, Spencer } \\
1990\end{array}$} \\
\hline Agromyzidae & $\begin{array}{l}\text { Liriomyza virgo } \\
\text { Zetterstedt }\end{array}$ & $\begin{array}{l}\text { Equisetum } \\
\text { palustre }\end{array}$ & $\begin{array}{l}\text { Nearctic and } \\
\text { Palaearctic Regions }\end{array}$ & \\
\hline Agromyzidae & Liriomyza virgula Frey ${ }^{8}$ & $\begin{array}{l}\text { Equisetum } \\
\text { arvense }\end{array}$ & Palearctic Regain & George 2014 \\
\hline Agromyzidae & $\begin{array}{l}\text { Phytoliriomyza clara } \\
\text { Melander }\end{array}$ & $\begin{array}{l}\text { Pteridium } \\
\text { aquilinum }\end{array}$ & Nearctic Region & $\begin{array}{l}\text { Eiseman 2020b, Spencer } \\
1990\end{array}$ \\
\hline Agromyzidae & $\begin{array}{l}\text { Phytoliriomyza } \\
\text { cyatheae Spencer }\end{array}$ & Cyathea dealbata & Neozelandic Region & \multirow[t]{2}{*}{$\begin{array}{l}\text { Spencer 1976, Spencer } \\
1990\end{array}$} \\
\hline Agromyzidae & $\begin{array}{l}\text { Phytoliriomyza } \\
\text { cyatheae Spencer }\end{array}$ & Cyathea smithii & Neozelandic Region & \\
\hline Agromyzidae & $\begin{array}{l}\text { Phytoliriomyza diplazii } \\
\text { Sasakawa }\end{array}$ & Diplazium & Oriental Region & Spencer 1990 \\
\hline Agromyzidae & $\begin{array}{l}\text { Phytoliriomyza felti } \\
\text { Malloch }\end{array}$ & $\begin{array}{l}\text { Asplenium } \\
\text { pinnatifidum }\end{array}$ & Nearctic Region & \multirow[t]{6}{*}{$\begin{array}{l}\text { Eiseman 2020b, Spencer } \\
1990\end{array}$} \\
\hline Agromyzidae & $\begin{array}{l}\text { Phytoliriomyza felti } \\
\text { Malloch }\end{array}$ & $\begin{array}{l}\text { Asplenium } \\
\text { platyneuron }\end{array}$ & Nearctic Region & \\
\hline Agromyzidae & $\begin{array}{l}\text { Phytoliriomyza felti } \\
\text { Malloch }\end{array}$ & $\begin{array}{l}\text { Pellaea } \\
\text { atropurpurea }\end{array}$ & Nearctic Region & \\
\hline Agromyzidae & $\begin{array}{l}\text { Phytoliriomyza felti } \\
\text { Malloch }\end{array}$ & Pellaea glabella & Nearctic Region & \\
\hline Agromyzidae & $\begin{array}{l}\text { Phytoliriomyza felti } \\
\text { Malloch }\end{array}$ & $\begin{array}{l}\text { Asplenium } \\
\text { rhizophyllum }\end{array}$ & Nearctic Region & \\
\hline Agromyzidae & $\begin{array}{l}\text { Phytoliriomyza felti } \\
\text { Malloch }\end{array}$ & Woodsia obtusa & Nearctic Region & \\
\hline Agromyzidae & $\begin{array}{l}\text { Phytoliriomyza } \\
\text { flavopleura Watt }\end{array}$ & Microsorum & Neozelandic Region & \multirow[t]{2}{*}{ Spencer 1990} \\
\hline Agromyzidae & $\begin{array}{l}\text { Phytoliriomyza } \\
\text { flavopleura Watt }\end{array}$ & $\begin{array}{l}\text { Notogrammitis } \\
\text { billardierei }\end{array}$ & Neozelandic Region & \\
\hline
\end{tabular}




\begin{tabular}{|c|c|c|c|c|}
\hline Miner family & Miner species & Host fern & $\begin{array}{l}\text { Biogeographical } \\
\text { regions }\end{array}$ & References \\
\hline Agromyzidae & $\begin{array}{l}\text { Phytoliriomyza } \\
\text { flavopleura Watt }\end{array}$ & $\begin{array}{l}\text { Asplenium } \\
\text { flaccidum }\end{array}$ & Neozelandic Region & \\
\hline Agromyzidae & $\begin{array}{l}\text { Phytoliriomyza } \\
\text { flavopleura Watt }\end{array}$ & $\begin{array}{l}\text { Asplenium } \\
\text { oblongifolium }\end{array}$ & Neozelandic Region & \\
\hline Agromyzidae & $\begin{array}{l}\text { Phytoliriomyza hilarella } \\
\text { Zetterstedt }\end{array}$ & Asplenium & Palaearctic Region & \multirow{3}{*}{$\begin{array}{l}\text { Ellis 2020, Lawton 1982, } \\
\text { Lawton 1976, MacGarvin } \\
\text { et al. 1986, Rigby and } \\
\text { Lawton 1981, Spencer } \\
1990\end{array}$} \\
\hline Agromyzidae & $\begin{array}{l}\text { Phytoliriomyza hilarella } \\
\text { Zetterstedt }\end{array}$ & $\begin{array}{l}\text { Pteridium } \\
\text { aquilinum }\end{array}$ & Palaearctic Region & \\
\hline Agromyzidae & $\begin{array}{l}\text { Phytoliriomyza hilarella } \\
\text { Zetterstedt }\end{array}$ & Polypodium & Palaearctic Region & \\
\hline Agromyzidae & $\begin{array}{l}\text { Phytoliriomyza kuscheli } \\
\text { Spencer }\end{array}$ & Histiopteris & Oriental Region & Spencer 1990 \\
\hline Agromyzidae & $\begin{array}{l}\text { Phytoliriomyza pteridii } \\
\text { Spencer }\end{array}$ & $\begin{array}{l}\text { Pteridium } \\
\text { aquilinum }\end{array}$ & Palaearctic Region & $\begin{array}{l}\text { Gerson 1979, Mcgavin } \\
\text { and Brown 1986, } \\
\text { MacGarvin et al. 1986, } \\
\text { Spencer } 1990\end{array}$ \\
\hline Agromyzidae & $\begin{array}{l}\text { Phytoliriomyza } \\
\text { pulchella Spencer* }\end{array}$ & $\begin{array}{l}\text { Pteridium } \\
\text { aquilinum }\end{array}$ & Nearctic Region & Eiseman 2020b \\
\hline Agromyzidae & Phytoliriomyza sp1 & Marattia* & Oriental Region & Spencer 1990 \\
\hline Agromyzidae & Phytoliriomyza sp2 & Cyathea & $\begin{array}{l}\text { Neotropical and } \\
\text { Andean Regions }\end{array}$ & Spencer 1990 \\
\hline Agromyzidae & $\begin{array}{l}\text { Phytoliriomyza } \\
\text { tearohensis Spencer }\end{array}$ & Cyathea dealbata & unknown & $\begin{array}{l}\text { Spencer 1976, Spencer } \\
1990\end{array}$ \\
\hline Agromyzidae & $\begin{array}{l}\text { Tropicomyia } \\
\text { angioptericola Shiao }\end{array}$ & $\begin{array}{l}\text { Angiopteris } \\
\text { lygodiifolia }\end{array}$ & Palaearctic Region & Shiao and Wu 2005 \\
\hline Agromyzidae & $\begin{array}{l}\text { Tropicomyia polyphaga } \\
\text { Spencer }\end{array}$ & Nephrolepis & Oriental Region & $\begin{array}{l}\text { Spencer 1990, Shiao and } \\
\text { Wu } 2005\end{array}$ \\
\hline Agromyzidae & Tropicomyia sp1 & Pleopeltis & Afrotopical Region & \multirow[t]{2}{*}{ Spencer 1990} \\
\hline Agromyzidae & Tropicomyia sp1 & $\begin{array}{l}\text { Asplenium } \\
\text { auriculatum }\end{array}$ & Afrotopical Region & \\
\hline Agromyzidae & Tropicomyia sp2 & $\begin{array}{l}\text { Angiopteris } \\
\text { evecta* }^{*}\end{array}$ & Oriental Region & Spencer 1990 \\
\hline Anthomyiidae & Chirosia albifrons Tiens & $\begin{array}{l}\text { Pteridium } \\
\text { aquilinum }\end{array}$ & Palaearctic Region & $\begin{array}{l}\text { Lawton 1976, MacGarvin } \\
\text { et al. } 1986\end{array}$ \\
\hline Anthomyiidae & $\begin{array}{l}\text { Chirosia albitarsis } \\
\text { Zetterstedt }\end{array}$ & $\begin{array}{l}\text { Pteridium } \\
\text { aquilinum }\end{array}$ & $\begin{array}{l}\text { Palaearctic and } \\
\text { Oriental Regions }\end{array}$ & $\begin{array}{l}\text { Ellis } 2020, \text { Gerson } 1979 \text {, } \\
\text { Lawton } 1976, \text { Mcgavin } \\
\text { and Brown 1986, Suwa } \\
\text { 1984, Suwa } 1999\end{array}$ \\
\hline Anthomyiidae & $\begin{array}{l}\text { Chirosia asperistilata } \\
\text { Suwa }\end{array}$ & $\begin{array}{l}\text { Dryopteris } \\
\text { monticola }\end{array}$ & Palaearctic Region & \multirow[t]{2}{*}{ Suwa 1999, Suwa 2005} \\
\hline Anthomyiidae & $\begin{array}{l}\text { Chirosia asperistilata } \\
\text { Suwa }\end{array}$ & $\begin{array}{l}\text { Dryopteris } \\
\text { crassirhizoma }\end{array}$ & Palaearctic Region & \\
\hline
\end{tabular}




\begin{tabular}{|c|c|c|c|c|}
\hline Miner family & Miner species & Host fern & $\begin{array}{l}\text { Biogeographical } \\
\text { regions }\end{array}$ & References \\
\hline Anthomyiidae & $\begin{array}{l}\text { Chirosia cinerosa } \\
\text { Zetterstedt }^{9}\end{array}$ & $\begin{array}{l}\text { Pteridium } \\
\text { aquilinum }\end{array}$ & Palaearctic Region & \multirow[t]{3}{*}{$\begin{array}{l}\text { Ellis 2020, Kwon and Suh } \\
\text { 1982, Suwa } 1999\end{array}$} \\
\hline Anthomyiidae & $\begin{array}{l}\text { Chirosia cinerosa } \\
\text { Zetterstedt }\end{array}$ & $\begin{array}{l}\text { Matteuccia } \\
\text { struthiopteris }\end{array}$ & Palaearctic Region & \\
\hline Anthomyiidae & $\begin{array}{l}\text { Chirosia cinerosa } \\
\text { Zetterstedt }\end{array}$ & $\begin{array}{l}\text { Athyrium filix- } \\
\text { femina }\end{array}$ & Palaearctic Region & \\
\hline Anthomyiidae & $\begin{array}{l}\text { Chirosia crassiseta } \\
\text { Stein }\end{array}$ & $\begin{array}{l}\text { Pteridium } \\
\text { aquilinum }\end{array}$ & Palaearctic Region & $\begin{array}{l}\text { Brown and McGavin 2007, } \\
\text { Ellis 2020, Gerson 1979, } \\
\text { Lawton 1976, Mcgavin } \\
\text { and Brown } 1986\end{array}$ \\
\hline Anthomyiidae & Chirosia filicis Huckett & $\begin{array}{l}\text { Osmunda } \\
\text { claytoniana }\end{array}$ & Nearctic Region & \multirow[t]{2}{*}{$\begin{array}{l}\text { Eiseman 2018, Eiseman } \\
\text { 2020b }\end{array}$} \\
\hline Anthomyiidae & Chirosia filicis Huckett & $\begin{array}{l}\text { Osmundastrum } \\
\text { cinnamomeum }\end{array}$ & Nearctic Region & \\
\hline Anthomyiidae & $\begin{array}{l}\text { Chirosia flavipennis } \\
\text { Fallen }\end{array}$ & $\begin{array}{l}\text { Pteridium } \\
\text { aquilinum }\end{array}$ & $\begin{array}{l}\text { Nearctic and } \\
\text { Palaearctic Regions }\end{array}$ & $\begin{array}{l}\text { Eiseman 2020b, Eiseman } \\
2018 \text {, Lawton 1976, Suwa } \\
2013\end{array}$ \\
\hline Anthomyiidae & $\begin{array}{l}\text { Chirosia gleniensis } \\
\text { Huckett }\end{array}$ & $\begin{array}{l}\text { Onoclea } \\
\text { sensibilis }\end{array}$ & Nearctic Region & \multirow[t]{3}{*}{$\begin{array}{l}\text { Eiseman 2020b, Eiseman } \\
\text { 2018, Eiseman 2020a }\end{array}$} \\
\hline Anthomyiidae & $\begin{array}{l}\text { Chirosia gleniensis } \\
\text { Huckett }\end{array}$ & Woodsia areolata & Nearctic Region & \\
\hline Anthomyiidae & $\begin{array}{l}\text { Chirosia gleniensis } \\
\text { Huckett }\end{array}$ & $\begin{array}{l}\text { Woodsia virginica } \\
\text { * }\end{array}$ & Nearctic Region & \\
\hline Anthomyiidae & $\begin{array}{l}\text { Chirosia griseifrons } \\
\text { Séguy }\end{array}$ & Dryopteris* & Palaearctic Region & \multirow[t]{2}{*}{ Ellis 2020, Suwa 1999} \\
\hline Anthomyiidae & $\begin{array}{l}\text { Chirosia griseifrons } \\
\text { Séguy }\end{array}$ & $\begin{array}{l}\text { Athyrium filix- } \\
\text { femina }\end{array}$ & Palaearctic Region & \\
\hline Anthomyiidae & $\begin{array}{l}\text { Chirosia grossicauda } \\
\text { Strobl }^{10}\end{array}$ & Asplenium & Palaearctic Region & \multirow{3}{*}{$\begin{array}{l}\text { Ellis } 2020, \text { Gerson } 1979 \text {, } \\
\text { Lawton } 1976 \text {, MacGarvin } \\
\text { et al. } 1986, \text { Mcgavin and } \\
\text { Brown } 1986 \text {, Suwa } 1999\end{array}$} \\
\hline Anthomyiidae & $\begin{array}{l}\text { Chirosia grossicauda } \\
\text { Strobl }\end{array}$ & $\begin{array}{l}\text { Pteridium } \\
\text { aquilinum }\end{array}$ & Palaearctic Region & \\
\hline Anthomyiidae & $\begin{array}{l}\text { Chirosia grossicauda } \\
\text { Strobl }\end{array}$ & Dryopteris* & Palaearctic Region & \\
\hline Anthomyiidae & $\begin{array}{l}\text { Chirosia histricina } \\
\text { Rondani }^{11}\end{array}$ & Osmunda regalis & $\begin{array}{l}\text { Nearctic and } \\
\text { Palaearctic Regions }\end{array}$ & \multirow{5}{*}{$\begin{array}{l}\text { Brown and McGavin 2007, } \\
\text { Ellis 2020, MacGarvin et } \\
\text { al. 1986, Mcgavin and } \\
\text { Brown 1986, Suwa } 1999\end{array}$} \\
\hline Anthomyiidae & $\begin{array}{l}\text { Chirosia histricina } \\
\text { Rondani }\end{array}$ & Asplenium & $\begin{array}{l}\text { Nearctic and } \\
\text { Palaearctic Regions }\end{array}$ & \\
\hline Anthomyiidae & $\begin{array}{l}\text { Chirosia histricina } \\
\text { Rondani }\end{array}$ & Blechnum spicant & $\begin{array}{l}\text { Nearctic and } \\
\text { Palaearctic Regions }\end{array}$ & \\
\hline Anthomyiidae & $\begin{array}{l}\text { Chirosia histricina } \\
\text { Rondani }\end{array}$ & $\begin{array}{l}\text { Pteridium } \\
\text { aquilinum }\end{array}$ & $\begin{array}{l}\text { Nearctic and } \\
\text { Palaearctic Regions }\end{array}$ & \\
\hline Anthomyiidae & $\begin{array}{l}\text { Chirosia histricina } \\
\text { Rondani }\end{array}$ & $\begin{array}{l}\text { Dryopteris filix- } \\
\text { mas }\end{array}$ & $\begin{array}{l}\text { Nearctic and } \\
\text { Palaearctic Regions }\end{array}$ & \\
\hline
\end{tabular}




\begin{tabular}{|c|c|c|c|c|}
\hline Miner family & Miner species & Host fern & $\begin{array}{l}\text { Biogeographical } \\
\text { regions }\end{array}$ & References \\
\hline Anthomyiidae & $\begin{array}{l}\text { Chirosia histricina } \\
\text { Rondani }\end{array}$ & $\begin{array}{l}\text { Matteuccia } \\
\text { struthiopteris }\end{array}$ & $\begin{array}{l}\text { Nearctic and } \\
\text { Palaearctic Regions }\end{array}$ & \\
\hline Anthomyiidae & $\begin{array}{l}\text { Chirosia histricina } \\
\text { Rondani }\end{array}$ & Polypodium & $\begin{array}{l}\text { Nearctic and } \\
\text { Palaearctic Regions }\end{array}$ & \\
\hline Anthomyiidae & $\begin{array}{l}\text { Chirosia histricina } \\
\text { Rondani }\end{array}$ & $\begin{array}{l}\text { Athyrium filix- } \\
\text { femina }\end{array}$ & $\begin{array}{l}\text { Nearctic and } \\
\text { Palaearctic Regions }\end{array}$ & \\
\hline Anthomyiidae & $\begin{array}{l}\text { Chirosia histricina } \\
\text { Rondani }\end{array}$ & $\begin{array}{l}\text { Cystopteris } \\
\text { fragilis }\end{array}$ & $\begin{array}{l}\text { Nearctic and } \\
\text { Palaearctic Regions }\end{array}$ & \\
\hline Anthomyiidae & $\begin{array}{l}\text { Chirosia montana } \\
\text { Pokorny }\end{array}$ & $\begin{array}{l}\text { Cystopteris } \\
\text { fragilis }\end{array}$ & $\begin{array}{l}\text { Nearctic and } \\
\text { Palaearctic Regions }\end{array}$ & $\begin{array}{l}\text { Eiseman 2020b, Eiseman } \\
2018\end{array}$ \\
\hline Anthomyiidae & Chirosia nigripes Bezzi & $\begin{array}{l}\text { Pteridium } \\
\text { aquilinum }\end{array}$ & Palaearctic Region & Ellis 2020, Suwa 1999 \\
\hline Anthomyiidae & $\begin{array}{l}\text { Chirosia pusillans } \\
\text { Huckett }\end{array}$ & $\begin{array}{l}\text { Athyrium filix- } \\
\text { femina }\end{array}$ & Nearctic Region & \multirow[t]{2}{*}{$\begin{array}{l}\text { Eiseman 2018, Eiseman } \\
\text { 2020a, Eiseman 2020b }\end{array}$} \\
\hline Anthomyiidae & $\begin{array}{l}\text { Chirosia pusillans } \\
\text { Huckett }\end{array}$ & $\begin{array}{l}\text { Matteuccia } \\
\text { struthiopteris }\end{array}$ & Nearctic Region & \\
\hline Anthomyiidae & $\begin{array}{l}\text { Chirosia spinosissima } \\
\text { Malloch }\end{array}$ & $\begin{array}{l}\text { Pteridium } \\
\text { aquilinum }\end{array}$ & $\begin{array}{l}\text { Nearctic and } \\
\text { Palaearctic Regions }\end{array}$ & $\begin{array}{l}\text { Eiseman } 2020 b \text {, Eiseman } \\
2020 a\end{array}$ \\
\hline Anthomyiidae & $\begin{array}{l}\text { Pegomya cedrica } \\
\text { Huckett }\end{array}$ & $\begin{array}{l}\text { Equisetum } \\
\text { hyemale }\end{array}$ & Nearctic Region & $\begin{array}{l}\text { Michelsen and Palmer } \\
2020\end{array}$ \\
\hline Anthomyiidae & $\begin{array}{l}\text { Pegomya disticha } \\
\text { Griffiths }\end{array}$ & $\begin{array}{l}\text { Equisetum } \\
\text { hyemale }\end{array}$ & Nearctic Region & $\begin{array}{l}\text { Michelsen and Palmer } \\
2020\end{array}$ \\
\hline Anthomyiidae & Pegomya glabra Stein & Equisetum & Nearctic Region & $\begin{array}{l}\text { Michelsen and Palmer } \\
2020\end{array}$ \\
\hline Drosophilidae & $\begin{array}{l}\text { Drosophila apicipuncta } \\
\text { Hardy }\end{array}$ & Sadleria & Nearctic Region & $\begin{array}{l}\text { Magnacca et al. } 2008, \\
\text { Magnacca and O'Grady } \\
2014 \text {, Maunsell et al. } 2016\end{array}$ \\
\hline Drosophilidae & $\begin{array}{l}\text { Drosophila diminuens } \\
\text { Hardy* }\end{array}$ & Sadleria & Nearctic Region & $\begin{array}{l}\text { Magnacca and O'Grady } \\
2014\end{array}$ \\
\hline Drosophilidae & $\begin{array}{l}\text { Drosophila sadleria } \\
\text { Bryan }\end{array}$ & Sadleria & Nearctic Region & Magnacca et al. 2008 \\
\hline Drosophilidae & $\begin{array}{l}\text { Scaptodrosophila notha } \\
\text { Bock }\end{array}$ & $\begin{array}{l}\text { Pteridium } \\
\text { aquilinum }\end{array}$ & $\begin{array}{l}\text { Australotropical and } \\
\text { Australotemperate } \\
\text { Regions }\end{array}$ & Maunsell et al. 2016 \\
\hline Drosophilidae & Scaptodrosophila sp. & $\begin{array}{l}\text { Parablechnum } \\
\text { wattsii }\end{array}$ & $\begin{array}{l}\text { Australotropical and } \\
\text { Australotemperate } \\
\text { Regions }\end{array}$ & Maunsell et al. 2016 \\
\hline Chironomidae & $\begin{array}{l}\text { Bryophaenocladius } \\
\text { furcatus Kieffer }\end{array}$ & Adiantum & $\begin{array}{l}\text { Nearctic and } \\
\text { Palaearctic Regions }\end{array}$ & Eiseman 2020b \\
\hline Pallopteridae & $\begin{array}{l}\text { Temnosira czurhini } \\
\text { Ozerov }\end{array}$ & Huperzia serrata & Palaearctic Region & Kato 2002 \\
\hline Lepidoptera & & & & \\
\hline
\end{tabular}




\begin{tabular}{|c|c|c|c|c|}
\hline Miner family & Miner species & Host fern & $\begin{array}{l}\text { Biogeographical } \\
\text { regions }\end{array}$ & References \\
\hline Crambidae & $\begin{array}{l}\text { Albusambia } \\
\text { elaphoglossumae Solis } \\
\text { \& Davis }\end{array}$ & $\begin{array}{l}\text { Elaphoglossum } \\
\text { conspersum }\end{array}$ & Nearctic Region & \multirow[t]{2}{*}{ Solis et al. $2005 a$} \\
\hline Crambidae & $\begin{array}{l}\text { Albusambia } \\
\text { elaphoglossumae Solis } \\
\text { \& Davis }\end{array}$ & $\begin{array}{l}\text { Elaphoglossum } \\
\text { biolleyi }\end{array}$ & Nearctic Region & \\
\hline Crambidae & $\begin{array}{l}\text { Eudonia zophoclaena } \\
\text { Meyrick }\end{array}$ & $\begin{array}{l}\text { Pyrrosia } \\
\text { eleagnifolia }\end{array}$ & Neozelandic Region & Patrick 2015 \\
\hline Crambidae & Scoparia illota Philpott & $\begin{array}{l}\text { Pyrrosia } \\
\text { eleagnifolia }\end{array}$ & Neozelandic Region & Patrick 2015 \\
\hline Crambidae & $\begin{array}{l}\text { Scoparia molifera } \\
\text { Meyrick }\end{array}$ & $\begin{array}{l}\text { Pyrrosia } \\
\text { eleagnifolia }\end{array}$ & Neozelandic Region & Patrick 2015 \\
\hline Crambidae & $\begin{array}{l}\text { Siamusotima aranea } \\
\text { Solis \& Yen }\end{array}$ & $\begin{array}{l}\text { Lygodium } \\
\text { flexuosum }\end{array}$ & Oriental Region & Solis et al. $2005 \mathrm{~b}$ \\
\hline Crambidae & $\begin{array}{l}\text { Siamusotima disrupta } \\
\text { Solis }\end{array}$ & Lygodium & Palaearctic Region & Solis et al. 2017 \\
\hline Crambidae & $\begin{array}{l}\text { Undulambia } \\
\text { polystichalis Capps }\end{array}$ & $\begin{array}{l}\text { Rumohra } \\
\text { adiantiformis }\end{array}$ & Nearctic Region & Gerson 1979 \\
\hline Noctuidae & $\begin{array}{l}\text { Hydraecia micacea } \\
\text { Esper }\end{array}$ & Equisetum & Palearctic Region & Ellis 2020 \\
\hline Noctuidae & $\begin{array}{l}\text { Papaipema inquaesita } \\
\text { Grote \& Robinson }\end{array}$ & $\begin{array}{l}\text { Onoclea } \\
\text { sensibilis }\end{array}$ & Nearctic Region & Bird 2012 \\
\hline Noctuidae & Papaipema pterisii Bird & $\begin{array}{l}\text { Pteridium } \\
\text { aquilinum }\end{array}$ & Nearctic Region & \multirow{2}{*}{$\begin{array}{l}\text { Bird 2012, Hinz and } \\
\text { Zahniser } 2015 \text { Schweitzer } \\
2012\end{array}$} \\
\hline Noctuidae & Papaipema pterisii Bird & $\begin{array}{l}\text { Matteuccia } \\
\text { struthiopteris* }^{*}\end{array}$ & Nearctic Region & \\
\hline Noctuidae & $\begin{array}{l}\text { Papaipema } \\
\text { speciosissima Grote \& } \\
\text { Robinson }\end{array}$ & Osmunda regalis & Nearctic Region & \multirow{2}{*}{$\begin{array}{l}\text { Hinz and Zahniser 2015, } \\
\text { Lafontaine and Schmidt } \\
2010, \text { Oppenheim et al. } \\
2018\end{array}$} \\
\hline Noctuidae & $\begin{array}{l}\text { Papaipema } \\
\text { speciosissima Grote \& } \\
\text { Robinson }\end{array}$ & $\begin{array}{l}\text { Osmundastrum } \\
\text { cinnamomeum }\end{array}$ & Nearctic Region & \\
\hline Noctuidae & $\begin{array}{l}\text { Papaipema stenocelis } \\
\text { Dyar }\end{array}$ & $\begin{array}{l}\text { Woodwardia } \\
\text { virginica }\end{array}$ & Nearctic Region & Chaloux, Andrea 2012 \\
\hline Noctuidae & $\begin{array}{l}\text { Pseudobryomima fallax } \\
\text { Hampson }\end{array}$ & $\begin{array}{l}\text { Pellaea } \\
\text { andromedifolia }\end{array}$ & Nearctic Region & Eiseman $2020 b$ \\
\hline Noctuidae & $\begin{array}{l}\text { Pseudobryomima } \\
\text { muscosa Hampson }\end{array}$ & $\begin{array}{l}\text { Polypodium } \\
\text { californicum }\end{array}$ & Nearctic Region & Eiseman $2020 \mathrm{~b}$ \\
\hline Tineidae & $\begin{array}{l}\text { Psychoides filicivora } \\
\text { Meyrick }^{12}\end{array}$ & $\begin{array}{l}\text { Asplenium } \\
\text { adiantum-nigrum }\end{array}$ & Palaearctic Region & $\begin{array}{l}\text { Gaedike 2019, Kim and } \\
\text { Bae } 2007\end{array}$ \\
\hline
\end{tabular}




\begin{tabular}{|c|c|c|c|c|}
\hline Miner family & Miner species & Host fern & $\begin{array}{l}\text { Biogeographical } \\
\text { regions }\end{array}$ & References \\
\hline Tineidae & $\begin{array}{l}\text { Psychoides filicivora } \\
\text { Meyrick }\end{array}$ & $\begin{array}{l}\text { Asplenium } \\
\text { ceterach }\end{array}$ & Palaearctic Region & \\
\hline Tineidae & $\begin{array}{l}\text { Psychoides filicivora } \\
\text { Meyrick }\end{array}$ & $\begin{array}{l}\text { Asplenium } \\
\text { scolopendrium }\end{array}$ & Palaearctic Region & \\
\hline Tineidae & $\begin{array}{l}\text { Psychoides filicivora } \\
\text { Meyrick }\end{array}$ & $\begin{array}{l}\text { Asplenium } \\
\text { trichomanes }\end{array}$ & Palaearctic Region & \\
\hline Tineidae & $\begin{array}{l}\text { Psychoides filicivora } \\
\text { Meyrick }\end{array}$ & $\begin{array}{l}\text { Dryopteris filix- } \\
\text { mas }\end{array}$ & Palaearctic Region & \\
\hline Tineidae & $\begin{array}{l}\text { Psychoides filicivora } \\
\text { Meyrick }\end{array}$ & $\begin{array}{l}\text { Dryopteris } \\
\text { aculeata }\end{array}$ & Palaearctic Region & \\
\hline Tineidae & $\begin{array}{l}\text { Psychoides filicivora } \\
\text { Meyrick }\end{array}$ & $\begin{array}{l}\text { Polystichum } \\
\text { setiferum }\end{array}$ & Palaearctic Region & \\
\hline Tineidae & $\begin{array}{l}\text { Psychoides gosari Kim } \\
\text { \& Bae }\end{array}$ & $\begin{array}{l}\text { Athyrium } \\
\text { yokoscense }\end{array}$ & $\begin{array}{l}\text { Oriental and } \\
\text { Palaearctic Regions }\end{array}$ & \multirow[t]{5}{*}{ Kim and Bae 2007} \\
\hline Tineidae & $\begin{array}{l}\text { Psychoides gosari Kim } \\
\text { \& Bae }\end{array}$ & Dryopteris setosa & $\begin{array}{l}\text { Oriental and } \\
\text { Palaearctic Region }\end{array}$ & \\
\hline Tineidae & $\begin{array}{l}\text { Psychoides gosari Kim } \\
\text { \& Bae }\end{array}$ & $\begin{array}{l}\text { Dryopteris } \\
\text { chinensis }\end{array}$ & $\begin{array}{l}\text { Oriental and } \\
\text { Palaearctic Regions }\end{array}$ & \\
\hline Tineidae & $\begin{array}{l}\text { Psychoides gosari Kim } \\
\text { \& Bae }\end{array}$ & $\begin{array}{l}\text { Dryopteris } \\
\text { crassirhizoma }\end{array}$ & $\begin{array}{l}\text { Oriental and } \\
\text { Palaearctic Regions }\end{array}$ & \\
\hline Tineidae & $\begin{array}{l}\text { Psychoides gosari Kim } \\
\text { \& Bae }\end{array}$ & $\begin{array}{l}\text { Dryopteris } \\
\text { saxifraga }\end{array}$ & $\begin{array}{l}\text { Oriental and } \\
\text { Palaearctic Regions }\end{array}$ & \\
\hline Tineidae & $\begin{array}{l}\text { Psychoides } \\
\text { phaedrospora Meyrick } \\
13\end{array}$ & Aspleniaceae & $\begin{array}{l}\text { Palaearctic and } \\
\text { Oriental Regions }\end{array}$ & $\begin{array}{l}\text { Gaedike } 2019, \text { Kim and } \\
\text { Bae } 2007\end{array}$ \\
\hline Tineidae & $\begin{array}{l}\text { Psychoides verhuella } \\
\text { Bruand }^{14}\end{array}$ & $\begin{array}{l}\text { Asplenium } \\
\text { ceterach }\end{array}$ & Palaearctic Region & \multirow{5}{*}{$\begin{array}{l}\text { Ellis } 2020, \text { Gaedike } 2019 \text {, } \\
\text { Heckford 2004, Kim and } \\
\text { Bae 2007, Muus } 2015\end{array}$} \\
\hline Tineidae & $\begin{array}{l}\text { Psychoides verhuella } \\
\text { Bruand }\end{array}$ & $\begin{array}{l}\text { Asplenium ruta- } \\
\text { muraria }\end{array}$ & Palaearctic Region & \\
\hline Tineidae & $\begin{array}{l}\text { Psychoides verhuella } \\
\text { Bruand }\end{array}$ & $\begin{array}{l}\text { Asplenium } \\
\text { scolopendrium }\end{array}$ & Palaearctic Region & \\
\hline Tineidae & $\begin{array}{l}\text { Psychoides verhuella } \\
\text { Bruand }\end{array}$ & $\begin{array}{l}\text { Asplenium } \\
\text { trichomanes }\end{array}$ & Palaearctic Region & \\
\hline Tineidae & $\begin{array}{l}\text { Psychoides verhuella } \\
\text { Bruand }\end{array}$ & $\begin{array}{l}\text { Pteridium } \\
\text { aquilinum }\end{array}$ & Palaearctic Region & \\
\hline Tortricidae & $\begin{array}{l}\text { Apoctena taipana } \\
\text { Felder \& Rogenhofer }\end{array}$ & $\begin{array}{l}\text { Pyrrosia } \\
\text { eleagnifolia }\end{array}$ & Neozelandic Region & Patrick 2015 \\
\hline Tortricidae & $\begin{array}{l}\text { Celypha tiedemanniana } \\
\text { Zeller }^{15}\end{array}$ & Equisetum & Palaearctic Region & Ellis 2020 \\
\hline Tortricidae & $\begin{array}{l}\text { Philocryptica polypodii } \\
\text { Watt }\end{array}$ & $\begin{array}{l}\text { Pyrrosia } \\
\text { eleagnifolia }\end{array}$ & Neozelandic Region & Patrick 2015 \\
\hline
\end{tabular}




\begin{tabular}{|c|c|c|c|c|}
\hline Miner family & Miner species & Host fern & $\begin{array}{l}\text { Biogeographical } \\
\text { regions }\end{array}$ & References \\
\hline Cosmopterigidae & $\begin{array}{l}\text { Hyposmocoma ( } \\
\text { Euperissus) ekaha } \\
\text { Swezey }\end{array}$ & Asplenium nidus & Oriental Region & Kawahara et al. 2011 \\
\hline Cosmopterigidae & $\begin{array}{l}\text { Hyposmocoma ( } \\
\text { Euperissus) trivitella } \\
\text { Swezey }\end{array}$ & $\begin{array}{l}\text { Elaphoglossum } \\
\text { aemulum }\end{array}$ & Oriental Region & \multirow[t]{4}{*}{ Kawahara et al. 2011} \\
\hline Cosmopterigidae & $\begin{array}{l}\text { Hyposmocoma ( } \\
\text { Euperissus) trivitella } \\
\text { Swezey }\end{array}$ & $\begin{array}{l}\text { Elaphoglossum } \\
\text { gorgoneum }\end{array}$ & Oriental Region & \\
\hline Cosmopterigidae & $\begin{array}{l}\text { Hyposmocoma ( } \\
\text { Euperissus) trivitella } \\
\text { Swezey }\end{array}$ & $\begin{array}{l}\text { Elaphoglossum } \\
\text { crassifolium }\end{array}$ & Oriental Region & \\
\hline Cosmopterigidae & $\begin{array}{l}\text { Hyposmocoma ( } \\
\text { Euperissus) trivitella } \\
\text { Swezey }\end{array}$ & $\begin{array}{l}\text { Elaphoglossum } \\
\text { reticulatum }\end{array}$ & Oriental Region & \\
\hline Gelechiidae & $\begin{array}{l}\text { Monochroa harrisonella } \\
\text { Busck }\end{array}$ & $\begin{array}{l}\text { Pteridium } \\
\text { aquilinum }\end{array}$ & Nearctic Region & Eiseman 2020b \\
\hline Gelechiidae & Paltodora cytisella Curti & $\begin{array}{l}\text { Pteridium } \\
\text { aquilinum }\end{array}$ & Palaearctic Region & $\begin{array}{l}\text { Lawton 1976, Rigby and } \\
\text { Lawton } 1981\end{array}$ \\
\hline Hepialidae & $\begin{array}{l}\text { Endoclita excrescens } \\
\text { Butler* }\end{array}$ & $\begin{array}{l}\text { Equisetum } \\
\text { arvense }\end{array}$ & Palaearctic Region & $\begin{array}{l}\text { Correia et al. 2020, } \\
\text { Grehan } 1989\end{array}$ \\
\hline Hepialidae & $\begin{array}{l}\text { Triodia sylvina } \\
\text { Linnaeus* }^{*}\end{array}$ & $\begin{array}{l}\text { Equisetum } \\
\text { arvense }\end{array}$ & Palaearctic Region & $\begin{array}{l}\text { Correia et al. 2020, } \\
\text { Grehan } 1989\end{array}$ \\
\hline Psychidae & $\begin{array}{l}\text { Apterona helicoidella } \\
\text { Vallot }\end{array}$ & Polypodium & unknown & Alders and Gielis 1999 \\
\hline \multicolumn{5}{|l|}{ Hymenoptera } \\
\hline Blasticotomidae & $\begin{array}{l}\text { Blasticotoma atra } \\
\text { Zhelochovtsev }\end{array}$ & unknown & unknown & $\begin{array}{l}\text { Taeger et al. } 2010 \text {, } \\
\text { Wikipedia } 2019\end{array}$ \\
\hline Blasticotomidae & $\begin{array}{l}\text { Blasticotoma filiceti } \\
\text { Klug }\end{array}$ & $\begin{array}{l}\text { Pteridium } \\
\text { aquilinum }\end{array}$ & Palaearctic Region & \multirow{6}{*}{$\begin{array}{l}\text { Ellis 2020, Liston } 2007 \text {, } \\
\text { Novgorodova and } \\
\text { Biryukova 2011, } \\
\text { Shcherbakov 2006, } \\
\text { Shcherbakov 2008, } \\
\text { Taeger et al. } 2010 \text {, } \\
\text { Wikipedia } 2019\end{array}$} \\
\hline Blasticotomidae & $\begin{array}{l}\text { Blasticotoma filiceti } \\
\text { Klug }\end{array}$ & Dryopteris & Palaearctic Region & \\
\hline Blasticotomidae & $\begin{array}{l}\text { Blasticotoma filiceti } \\
\text { Klug }\end{array}$ & Polystichum & Palaearctic Region & \\
\hline Blasticotomidae & $\begin{array}{l}\text { Blasticotoma filiceti } \\
\text { Klug }\end{array}$ & $\begin{array}{l}\text { Matteuccia } \\
\text { struthiopteris }\end{array}$ & Palaearctic Region & \\
\hline Blasticotomidae & $\begin{array}{l}\text { Blasticotoma filiceti } \\
\text { Klug }\end{array}$ & Athyrium alpestre & Palaearctic Region & \\
\hline Blasticotomidae & $\begin{array}{l}\text { Blasticotoma filiceti } \\
\text { Klug }\end{array}$ & $\begin{array}{l}\text { Athyrium filix- } \\
\text { femina }\end{array}$ & Palaearctic Region & \\
\hline Blasticotomidae & $\begin{array}{l}\text { Blasticotoma filiceti var. } \\
\text { pacificus Malaise }\end{array}$ & unknown & unknown & $\begin{array}{l}\text { Taeger et al. } 2010, \\
\text { Wikipedia } 2019\end{array}$ \\
\hline
\end{tabular}




\begin{tabular}{|c|c|c|c|c|}
\hline Miner family & Miner species & Host fern & $\begin{array}{l}\text { Biogeographical } \\
\text { regions }\end{array}$ & References \\
\hline Blasticotomidae & $\begin{array}{l}\text { Blasticotoma nipponica } \\
\text { Takeuchi }\end{array}$ & unknown & unknown & $\begin{array}{l}\text { Wikipedia 2019, Taeger et } \\
\text { al. } 2010\end{array}$ \\
\hline Blasticotomidae & $\begin{array}{l}\text { Blasticotoma smithi } \\
\text { Shinohara }\end{array}$ & unknown & unknown & $\begin{array}{l}\text { Taeger et al. } 2010, \\
\text { Wikipedia } 2019\end{array}$ \\
\hline Blasticotomidae & $\begin{array}{l}\text { Blasticotoma warabii } \\
\text { Togashi }\end{array}$ & unknown & unknown & $\begin{array}{l}\text { Taeger et al. } 2010, \\
\text { Wikipedia } 2019\end{array}$ \\
\hline Blasticotomidae & Bohea abrupta Maa & unknown & unknown & $\begin{array}{l}\text { Taeger et al. } 2010, \\
\text { Wikipedia } 2019\end{array}$ \\
\hline Blasticotomidae & $\begin{array}{l}\text { Paremphytus ostentus } \\
\text { Brues }\end{array}$ & unknown & unknown & $\begin{array}{l}\text { Taeger et al. } 2010, \\
\text { Wikipedia } 2019\end{array}$ \\
\hline Blasticotomidae & $\begin{array}{l}\text { Runaria flavipes } \\
\text { Takeuchi }\end{array}$ & unknown & unknown & $\begin{array}{l}\text { Taeger et al. } 2010, \\
\text { Wikipedia } 2019\end{array}$ \\
\hline Blasticotomidae & $\begin{array}{l}\text { Runaria hunannica Wei } \\
\text { in Wei \& Nie }\end{array}$ & unknown & unknown & $\begin{array}{l}\text { Taeger et al. } 2010, \\
\text { Wikipedia } 2019\end{array}$ \\
\hline Blasticotomidae & $\begin{array}{l}\text { Runaria punctata Wei } \\
\text { in Wei \& Nie }\end{array}$ & unknown & unknown & $\begin{array}{l}\text { Taeger et al. } 2010, \\
\text { Wikipedia } 2019\end{array}$ \\
\hline Blasticotomidae & $\begin{array}{l}\text { Runaria shaanxinica } \\
\text { Wei in Wei \& Nie }\end{array}$ & unknown & unknown & $\begin{array}{l}\text { Taeger et al. } 2010, \\
\text { Wikipedia } 2019\end{array}$ \\
\hline Blasticotomidae & $\begin{array}{l}\text { Runaria taiwana } \\
\text { Shinohara }\end{array}$ & unknown & unknown & $\begin{array}{l}\text { Taeger et al. } 2010, \\
\text { Wikipedia } 2019\end{array}$ \\
\hline Tenthredinidae & $\begin{array}{l}\text { Aneugmenus coronatus } \\
\text { Klug }\end{array}$ & $\begin{array}{l}\text { Pteridium } \\
\text { aquilinum }\end{array}$ & Palaearctic Region & \multirow[t]{4}{*}{$\begin{array}{l}\text { Beneš 2014, Ellis 2020, } \\
\text { Schwarz } 2005\end{array}$} \\
\hline Tenthredinidae & $\begin{array}{l}\text { Aneugmenus coronatus } \\
\text { Klug }\end{array}$ & $\begin{array}{l}\text { Dryopteris filix- } \\
\text { mas }\end{array}$ & Palaearctic Region & \\
\hline Tenthredinidae & $\begin{array}{l}\text { Aneugmenus coronatus } \\
\text { Klug }\end{array}$ & $\begin{array}{l}\text { Polystichum } \\
\text { setiferum }\end{array}$ & Palaearctic Region & \\
\hline Tenthredinidae & $\begin{array}{l}\text { Aneugmenus coronatus } \\
\text { Klug }\end{array}$ & $\begin{array}{l}\text { Athyrium filix- } \\
\text { femina }\end{array}$ & Palaearctic Region & \\
\hline Tenthredinidae & $\begin{array}{l}\text { Heptamelus dahlbomi } \\
\text { Thomson }\end{array}$ & $\begin{array}{l}\text { Athyrium filix- } \\
\text { femina }\end{array}$ & $\begin{array}{l}\text { Nearctic and } \\
\text { Palaearctic Regions }\end{array}$ & Vikberg and Liston 2009 \\
\hline Tenthredinidae & $\begin{array}{l}\text { Heptamelus } \\
\text { ochroleucus Stephens } \\
16\end{array}$ & Blechnum spicant & $\begin{array}{l}\text { Nearctic and } \\
\text { Palaearctic Regions }\end{array}$ & \multirow[t]{5}{*}{$\begin{array}{l}\text { Ellis 2020, Shcherbakov } \\
2008, \text { Vikberg } 2017, \\
\text { Vikberg and Liston } 2009\end{array}$} \\
\hline Tenthredinidae & $\begin{array}{l}\text { Heptamelus } \\
\text { ochroleucus Stephens }\end{array}$ & $\begin{array}{l}\text { Matteuccia } \\
\text { struthiopteris }\end{array}$ & $\begin{array}{l}\text { Nearctic and } \\
\text { Palaearctic Regions }\end{array}$ & \\
\hline Tenthredinidae & $\begin{array}{l}\text { Heptamelus } \\
\text { ochroleucus Stephens }\end{array}$ & $\begin{array}{l}\text { Dryopteris } \\
\text { dilatata }\end{array}$ & $\begin{array}{l}\text { Nearctic and } \\
\text { Palaearctic Regions }\end{array}$ & \\
\hline Tenthredinidae & $\begin{array}{l}\text { Heptamelus } \\
\text { ochroleucus Stephens }\end{array}$ & $\begin{array}{l}\text { Polypodium } \\
\text { vulgare }\end{array}$ & $\begin{array}{l}\text { Nearctic and } \\
\text { Palaearctic Regions }\end{array}$ & \\
\hline Tenthredinidae & $\begin{array}{l}\text { Heptamelus } \\
\text { ochroleucus Stephens }\end{array}$ & $\begin{array}{l}\text { Athyrium filix- } \\
\text { femina }\end{array}$ & $\begin{array}{l}\text { Nearctic and } \\
\text { Palaearctic Regions }\end{array}$ & \\
\hline Coleoptera & & & & \\
\hline
\end{tabular}




\begin{tabular}{|c|c|c|c|c|}
\hline Miner family & Miner species & Host fern & $\begin{array}{l}\text { Biogeographical } \\
\text { regions }\end{array}$ & References \\
\hline Buprestidae & $\begin{array}{l}\text { Endelus bakerianus } \\
\text { Obenberger }\end{array}$ & $\begin{array}{l}\text { Lygodium } \\
\text { microphyllum }\end{array}$ & Oriental Region & $\begin{array}{l}\text { Kalashian } 2013 \text {, Goolsby } \\
\text { et al. } 2003 \text {, Mehltreter et } \\
\text { al. } 2010\end{array}$ \\
\hline Buprestidae & $\begin{array}{l}\text { Neotrachys bellamyi } \\
\text { Hespenheide }\end{array}$ & Gleichenia glauca & Neotropical Region & Hespenheide 2006 \\
\hline Buprestidae & $\begin{array}{l}\text { Neotrachys bicolor } \\
\text { Hespenheide }\end{array}$ & $\begin{array}{l}\text { Cnemidaria } \\
\text { petiolata }\end{array}$ & Neotropical Region & Hespenheide 1982 \\
\hline Buprestidae & $\begin{array}{l}\text { Neotrachys bordoni } \\
\text { Cobos }\end{array}$ & Cyatheaceae & Neotropical Region & Hespenheide 1982 \\
\hline Buprestidae & $\begin{array}{l}\text { Neotrachys caerulea } \\
\text { Hespenheide }\end{array}$ & Cyatheaceae & Neotropical Region & Hespenheide 1982 \\
\hline Buprestidae & $\begin{array}{l}\text { Neotrachys concinna } \\
\text { Fisher }\end{array}$ & Cyatheaceae & Neotropical Region & $\begin{array}{l}\text { Hespenheide 1982, } \\
\text { Hespenheide } 2006\end{array}$ \\
\hline Buprestidae & $\begin{array}{l}\text { Neotrachys } \\
\text { cyanipennis Fisher }\end{array}$ & Cyatheaceae & Neotropical Region & Hespenheide 2006 \\
\hline Buprestidae & $\begin{array}{l}\text { Neotrachys estebana } \\
\text { Kerremans* }\end{array}$ & Dicranopteris & Neotropical Region & Hespenheide 1982 \\
\hline Buprestidae & $\begin{array}{l}\text { Neotrachys fennahi } \\
\text { Thery }\end{array}$ & Cyatheaceae & Neotropical Region & Hespenheide 1980 \\
\hline Buprestidae & $\begin{array}{l}\text { Neotrachys gleicheniae } \\
\text { Hespenheide }\end{array}$ & Gleichenia & Neotropical Region & Hespenheide 1982 \\
\hline Buprestidae & $\begin{array}{l}\text { Neotrachys hoffmani } \\
\text { Fisher }\end{array}$ & Cyatheaceae & Neotropical Region & $\begin{array}{l}\text { Hespenheide 1980, } \\
\text { Hespenheide } 1982\end{array}$ \\
\hline Buprestidae & $\begin{array}{l}\text { Neotrachys mariae } \\
\text { Hespenheide }\end{array}$ & Gleichenia & Neotropical Region & Hespenheide 2006 \\
\hline Buprestidae & $\begin{array}{l}\text { Neotrachys } \\
\text { resplendens } \\
\text { Hespenheide }\end{array}$ & Cyatheaceae & Neotropical Region & Hespenheide 1982 \\
\hline Buprestidae & $\begin{array}{l}\text { Neotrachys segregata } \\
\text { Waterhouse }\end{array}$ & Gleicheniaceae* & Neotropical Region & Hespenheide 1982 \\
\hline Buprestidae & $\begin{array}{l}\text { Neotrachys solisi } \\
\text { Hespenheide }\end{array}$ & Gleichenia & Neotropical Region & Hespenheide 2006 \\
\hline Chrysomelidae & Febra insularis Bryant & $\begin{array}{l}\text { Acrostichum } \\
\text { aureum }\end{array}$ & Oriental Region & $\begin{array}{l}\text { Samuelson 1973, } \\
\text { Santiago-Blay } 2004\end{array}$ \\
\hline Chrysomelidae & Febra ovata Bryant & $\begin{array}{l}\text { Angiopteris } \\
\text { evecta }\end{array}$ & Oriental Region & $\begin{array}{l}\text { Samuelson } 1973, \text { Nadein } \\
\text { 2013, Jolivet } 1991\end{array}$ \\
\hline
\end{tabular}




\begin{tabular}{|c|c|c|c|c|}
\hline Miner family & Miner species & Host fern & $\begin{array}{l}\text { Biogeographical } \\
\text { regions }\end{array}$ & References \\
\hline Chrysomelidae & Febra venusta Clark & Nephrolepis & Oriental Region & $\begin{array}{l}\text { Samuelson 1973, } \\
\text { Santiago-Blay } 2004\end{array}$ \\
\hline Chrysomelidae & $\begin{array}{l}\text { Halticorcus bhaumiki } \\
\text { Basu et Sengupta }^{17}\end{array}$ & Pteris vittata & $\begin{array}{l}\text { Palaearctic and } \\
\text { Oriental Regions }\end{array}$ & \multirow{10}{*}{$\begin{array}{l}\text { Isowa and Kojima } 2011 \text {, } \\
\text { Konstantinov and } \\
\text { Prathapan 2008, Patra } \\
\text { and Bera } 2007\end{array}$} \\
\hline Chrysomelidae & $\begin{array}{l}\text { Halticorcus bhaumiki } \\
\text { Basu et Sengupta }\end{array}$ & $\begin{array}{l}\text { Ampelopteris } \\
\text { prolifera }\end{array}$ & $\begin{array}{l}\text { Palaearctic and } \\
\text { Oriental Regions }\end{array}$ & \\
\hline Chrysomelidae & $\begin{array}{l}\text { Halticorcus bhaumiki } \\
\text { Basu et Sengupta }\end{array}$ & Cyclosorus & $\begin{array}{l}\text { Palaearctic and } \\
\text { Oriental Regions }\end{array}$ & \\
\hline Chrysomelidae & $\begin{array}{l}\text { Halticorcus bhaumiki } \\
\text { Basu et Sengupta }\end{array}$ & Christella dentata & $\begin{array}{l}\text { Palaearctic and } \\
\text { Oriental Regions }\end{array}$ & \\
\hline Chrysomelidae & $\begin{array}{l}\text { Halticorcus bhaumiki } \\
\text { Basu et Sengupta }\end{array}$ & $\begin{array}{l}\text { Nephrolepis } \\
\text { cordifolia }\end{array}$ & $\begin{array}{l}\text { Palaearctic and } \\
\text { Oriental Regions }\end{array}$ & \\
\hline Chrysomelidae & $\begin{array}{l}\text { Halticorcus bhaumiki } \\
\text { Basu et Sengupta }\end{array}$ & $\begin{array}{l}\text { Nephrolepis } \\
\text { exaltata }\end{array}$ & $\begin{array}{l}\text { Palaearctic and } \\
\text { Oriental Regions }\end{array}$ & \\
\hline Chrysomelidae & $\begin{array}{l}\text { Halticorcus bhaumiki } \\
\text { Basu et Sengupta }\end{array}$ & $\begin{array}{l}\text { Adiantum } \\
\text { lunulatum }\end{array}$ & $\begin{array}{l}\text { Palaearctic and } \\
\text { Oriental Regions }\end{array}$ & \\
\hline Chrysomelidae & $\begin{array}{l}\text { Halticorcus bhaumiki } \\
\text { Basu et Sengupta }\end{array}$ & $\begin{array}{l}\text { Drynaria } \\
\text { propinqua }\end{array}$ & $\begin{array}{l}\text { Palaearctic and } \\
\text { Oriental Regions }\end{array}$ & \\
\hline Chrysomelidae & $\begin{array}{l}\text { Halticorcus bhaumiki } \\
\text { Basu et Sengupta }\end{array}$ & $\begin{array}{l}\text { Pyrrosia } \\
\text { adnascens }\end{array}$ & $\begin{array}{l}\text { Palaearctic and } \\
\text { Oriental Regions }\end{array}$ & \\
\hline Chrysomelidae & $\begin{array}{l}\text { Halticorcus bhaumiki } \\
\text { Basu et Sengupta }\end{array}$ & $\begin{array}{l}\text { Microsorum } \\
\text { scolopendria }\end{array}$ & $\begin{array}{l}\text { Palaearctic and } \\
\text { Oriental Regions }\end{array}$ & \\
\hline Chrysomelidae & $\begin{array}{l}\text { Halticorcus hiranoi } \\
\text { Takizawa }^{18}\end{array}$ & $\begin{array}{l}\text { Lemmaphyllum } \\
\text { microphyllum }\end{array}$ & Palaearctic Region & \multirow[t]{2}{*}{$\begin{array}{l}\text { Kato 1991, Santiago-Blay } \\
2004\end{array}$} \\
\hline Chrysomelidae & $\begin{array}{l}\text { Halticorcus hiranoi } \\
\text { Takizawa }\end{array}$ & $\begin{array}{l}\text { Loxogramme } \\
\text { salicifolia* }\end{array}$ & Palaearctic Region & \\
\hline Chrysomelidae & $\begin{array}{l}\text { Halticorcus kasuga } \\
\text { Nakane }\end{array}$ & $\begin{array}{l}\text { Lepisorus } \\
\text { thunbergianus }\end{array}$ & Palaearctic Region & \multirow[t]{4}{*}{ Isowa and Kojima 2011} \\
\hline Chrysomelidae & $\begin{array}{l}\text { Halticorcus kasuga } \\
\text { Nakane }\end{array}$ & Lepisorus onoei & Palaearctic Region & \\
\hline Chrysomelidae & $\begin{array}{l}\text { Halticorcus kasuga } \\
\text { Nakane }\end{array}$ & $\begin{array}{l}\text { Lemmaphyllum } \\
\text { microphyllum }\end{array}$ & Palaearctic Region & \\
\hline Chrysomelidae & $\begin{array}{l}\text { Halticorcus kasuga } \\
\text { Nakane }\end{array}$ & $\begin{array}{l}\text { Pyrrosia } \\
\text { linearifolia }\end{array}$ & Palaearctic Region & \\
\hline Chrysomelidae & $\begin{array}{l}\text { Halticorcus platycerii } \\
\text { Lea }\end{array}$ & $\begin{array}{l}\text { Platycerium } \\
\text { alcicorne }\end{array}$ & $\begin{array}{l}\text { Australotropical, } \\
\text { Australotemperate and } \\
\text { Palaearctic Regions }\end{array}$ & \multirow[t]{2}{*}{$\begin{array}{l}\text { Hawkeswood 2003, Isowa } \\
\text { and Kojima 2011, Sinclair } \\
\text { and Hughes } 2010\end{array}$} \\
\hline Chrysomelidae & $\begin{array}{l}\text { Halticorcus platycerii } \\
\text { Lea }\end{array}$ & Asplenium nidus & $\begin{array}{l}\text { Australotropical, } \\
\text { Australotemperate and } \\
\text { Palaearctic Regions }\end{array}$ & \\
\hline Chrysomelidae & $\begin{array}{l}\text { Halticorcus sauteri } \\
\text { Chen }{ }^{19}\end{array}$ & Colysis elliptica & Palaearctic Region & $\begin{array}{l}\text { Kato 1991, Santiago-Blay } \\
2004\end{array}$ \\
\hline
\end{tabular}




\begin{tabular}{|c|c|c|c|c|}
\hline Miner family & Miner species & Host fern & $\begin{array}{l}\text { Biogeographical } \\
\text { regions }\end{array}$ & References \\
\hline Chrysomelidae & $\begin{array}{l}\text { Halticorcus sauteri } \\
\text { Chen }\end{array}$ & $\begin{array}{l}\text { Leptochilus } \\
\text { ellipticus }\end{array}$ & Palaearctic Region & \\
\hline Chrysomelidae & $\begin{array}{l}\text { Hippuriphila babai } \\
\text { Chujo* }\end{array}$ & Equisetum & Palaearctic Region & $\begin{array}{l}\text { Correia et al. 2020, Poinar } \\
2014\end{array}$ \\
\hline Chrysomelidae & $\begin{array}{l}\text { Hippuriphila } \\
\text { canadensis Brown* }\end{array}$ & $\begin{array}{l}\text { Equisetum } \\
\text { arvense }\end{array}$ & Nearctic Region & $\begin{array}{l}\text { Correia et al. 2020, Poinar } \\
2014\end{array}$ \\
\hline Chrysomelidae & $\begin{array}{l}\text { Hippuriphila catherinae } \\
\text { Barr* }\end{array}$ & Equisetum & Neotropical Region & $\begin{array}{l}\text { Correia et al. 2020, Poinar } \\
2014\end{array}$ \\
\hline Chrysomelidae & $\begin{array}{l}\text { Hippuriphila equiseti } \\
\text { Beller et Hatch* }\end{array}$ & $\begin{array}{l}\text { Equisetum } \\
\text { arvense }\end{array}$ & Nearctic Region & $\begin{array}{l}\text { Correia et al. 2020, Poinar } \\
2014\end{array}$ \\
\hline Chrysomelidae & $\begin{array}{l}\text { Hippuriphila modeeri } \\
\text { Linnaeus }\end{array}$ & $\begin{array}{l}\text { Equisetum } \\
\text { arvense }\end{array}$ & Palaearctic Region & \multirow{3}{*}{$\begin{array}{l}\text { Biological Records Centre } \\
\text { 2020, Ellis 2020, } \\
\text { Santiago-Blay } 2004\end{array}$} \\
\hline Chrysomelidae & $\begin{array}{l}\text { Hippuriphila modeeri } \\
\text { Linnaeus }\end{array}$ & $\begin{array}{l}\text { Equisetum } \\
\text { fluviatile }\end{array}$ & Palaearctic Region & \\
\hline Chrysomelidae & $\begin{array}{l}\text { Hippuriphila modeeri } \\
\text { Linnaeus }\end{array}$ & $\begin{array}{l}\text { Equisetum } \\
\text { palustre }\end{array}$ & Palaearctic Region & \\
\hline Curculionidae & $\begin{array}{l}\text { Bagous claudicans } \\
\text { Boheman }\end{array}$ & $\begin{array}{l}\text { Equisetum } \\
\text { fluviatile }\end{array}$ & $\begin{array}{l}\text { except for Central and } \\
\text { South America, all the } \\
\text { world }\end{array}$ & $\begin{array}{l}\text { Ellis } 2020, \text { Gosik et al. } \\
2019\end{array}$ \\
\hline Curculionidae & $\begin{array}{l}\text { Bagous lutulentus } \\
\text { Gyllenhal }^{20}\end{array}$ & $\begin{array}{l}\text { Equisetum } \\
\text { fluviatile }\end{array}$ & $\begin{array}{l}\text { except for Central and } \\
\text { South America, all the } \\
\text { world }\end{array}$ & $\begin{array}{l}\text { Ellis 2020, Gosik 2009, } \\
\text { Gosik et al. 2019, Gosik } \\
\text { and Wanat } 2014\end{array}$ \\
\hline Curculionidae & $\begin{array}{l}\text { Grypus brunnirostris } \\
\text { Fabricius }^{21}\end{array}$ & $\begin{array}{l}\text { Equisetum } \\
\text { arvense }\end{array}$ & Nearctic Region & \multirow[t]{3}{*}{ Ellis 2020, George 2014} \\
\hline Curculionidae & $\begin{array}{l}\text { Grypus brunnirostris } \\
\text { Fabricius }\end{array}$ & $\begin{array}{l}\text { Equisetum } \\
\text { fluviatile }\end{array}$ & Nearctic Region & \\
\hline Curculionidae & $\begin{array}{l}\text { Grypus brunnirostris } \\
\text { Fabricius }\end{array}$ & $\begin{array}{l}\text { Equisetum } \\
\text { ramosissimum }\end{array}$ & Nearctic Region & \\
\hline Curculionidae & $\begin{array}{l}\text { Grypus equiseti } \\
\text { Fabricius }^{22}\end{array}$ & $\begin{array}{l}\text { Equisetum } \\
\text { arvense }\end{array}$ & $\begin{array}{l}\text { Nearctic and } \\
\text { Palaearctic Regions }\end{array}$ & \multirow[t]{4}{*}{$\begin{array}{l}\text { Ellis 2020, George 2014, } \\
\text { Gosik et al. } 2019\end{array}$} \\
\hline Curculionidae & $\begin{array}{l}\text { Grypus equiseti } \\
\text { Fabricius }\end{array}$ & $\begin{array}{l}\text { Equisetum } \\
\text { palustre }\end{array}$ & $\begin{array}{l}\text { Nearctic and } \\
\text { Palaearctic Regions }\end{array}$ & \\
\hline Curculionidae & $\begin{array}{l}\text { Grypus equiseti } \\
\text { Fabricius }\end{array}$ & $\begin{array}{l}\text { Equisetum } \\
\text { pratense }\end{array}$ & $\begin{array}{l}\text { Nearctic and } \\
\text { Palaearctic Regions }\end{array}$ & \\
\hline Curculionidae & $\begin{array}{l}\text { Grypus equiseti } \\
\text { Fabricius }\end{array}$ & $\begin{array}{l}\text { Equisetum } \\
\text { sylvaticum }\end{array}$ & $\begin{array}{l}\text { Nearctic and } \\
\text { Palaearctic Regions }\end{array}$ & \\
\hline Curculionidae & $\begin{array}{l}\text { Stenopelmus rufinasus } \\
\text { Gyllenhal }\end{array}$ & Azolla & $\begin{array}{l}\text { Nearctic, Afrotopical } \\
\text { and Palaearctic } \\
\text { Regions }\end{array}$ & $\begin{array}{l}\text { Center et al. 2002, Hill } \\
\text { and Cilliers 1999, } \\
\text { Richerson and Grigarick } \\
1967\end{array}$ \\
\hline
\end{tabular}

\footnotetext{
* possible host fern or miner
} 
Synonyms:

1 Chromatomyia cheilanthus Garg = Phytomyza cheilanthus Garg;

2 Chromatomyia dorsata Hendel = Phytomyza dorsata Hendel;

3 Chromatomyia dryoptericola Sasakawa = Phytomyza dryoptericola Sasakawa

4 Chromatomyia scolopendri Robineau-Desvoidy = Phytomyza scolopendri Goureau = Phytomyza elegans Goureau = Phytomyza nevadensis Strobl = Chromatoinyia nevadensis Strobl;

5 Liriomyza equiseti de Meijere = Liriomyza kenti Spencer;

6 Liriomyza occipitalis Hendel = Liriomyza bruscae Hering;

7 Liriomyza virgo Zetterstedt $=$ Liriomyza arcticola Spencer $=$ Phytomyza pallicornis Zetterstedt;

8 Liriomyza virgula Frey = Liriomyza larissa Hering;

9 Chirosia cinerosa Zetterstedt $=$ Pycnoglossa cinerosa Zetterstedt;

10 Chirosia grossicauda Strobl = Chirosia parvicornis nec Zetterstedt;

11 Chirosia histricina Rondani $=$ Chirosia setifemur Ringdahl $=$ Pycnoglossa hystrix Brischke $=$ Pycnoglossa hystricina;

12 Psychoides filicivora Meyrick = Teichobia filicivora Meyrick;

13 Psychoides phaedrospora Meyrick = Mnesipatris phadrospora Meyrick;

14 Psychoides verhuella Bruand = Teichobia verhuellella Herrich-Schaffer = Lambrosetia verhuellella Stainton;

15 Celypha tiedemanniana Zeller = Olethreutes tiedemanniana Zeller Kuznetsov = Syricoris tiedemanniana Zeller;

16 Heptamelus ochroleucus Stephens = Melicerta ochroleucus Stephens;

17 Halticorcus bhaumiki Basu et Sengupta = Schenklingia bhaumiki Basu and Sengupta;

18 Halticorcus hiranoi Takizawa = Schenklingia hiranoi Takizawa;

19 Halticorcus sauteri Chen = Schenklingia sauteri Chen;

20 Bagous lutulentus Gyllenhal = Bagous glabrirostris var. nigritarsis Thomson;

21 Grypus brunnirostris Fabricius = Curculio Inspectionatus Herbst;

22 Grypus equiseti Fabricius = Grypidius equiseti Fabricius;

Fern miners belong to four orders: Diptera (51 species; 39.8\%), Coleoptera (33 species; 25.8\%), Lepidoptera (28 species; $21.9 \%$ ) and Hymenoptera (16 species; 12.5\%) (Fig. 1; Suppl. material 2). In general, dipteran leaf miners are dominant in herbaceous plants while lepidopteran leaf miners are dominant in woody plants (De Prins and De Prins 2020, Edmunds et al. 2020, Eiseman 2020b, Ellis 2020, Pitkin et al. 2019, Spencer 1990). The life form of most extant ferns is herbaceous, which could explain why nearly half of fernmining species are dipteran flies. 


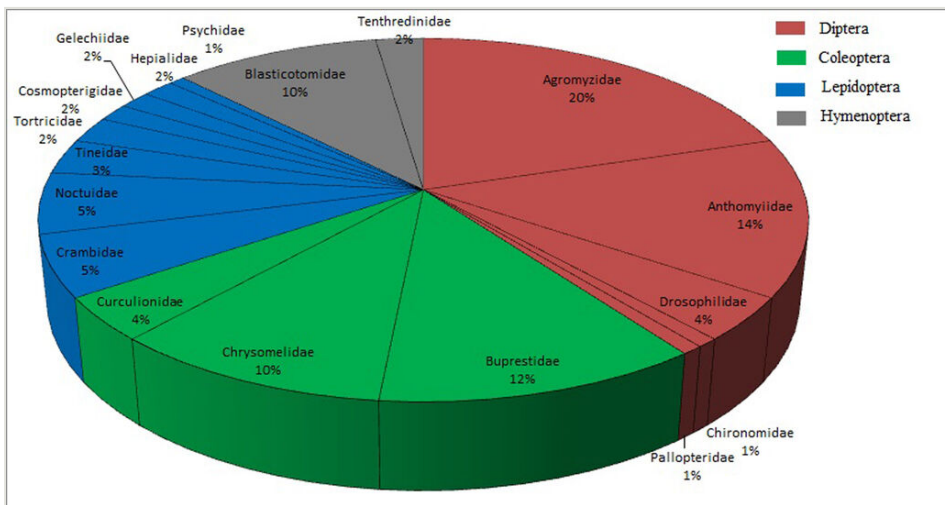

Figure 1. doi

Percentage distribution of the fern-mining species into the four orders and the 18 families.

Amongst the 18 fern-mining insect families, Agromyzidae, Anthomyiidae, Buprestidae, Chrysomelidae and Blasticotomidae had the highest numbers of species $(20.3 \%, 14.1 \%$, $11.7 \%, 10.2 \%$ and $10.2 \%$, respectively), while the other 13 families accounted for $33.5 \%$ only (Fig. 1; Suppl. material 2).

The fern families with highest numbers of host species were Dryopteridaceae (19), Polypodiaceae (18) and Aspleniaceae (15) (Fig. 2; Suppl. material 3). The fern families with the highest numbers of miner species were Dennstaedtiaceae (21), Equisetaceae (21), Polypodiaceae (20) and Aspleniaceae (14) (Fig. 2; Suppl. material 3). With 82 species and 12 families of host ferns and 67 species of fern miners, Polypodiales was the dominant host order of fern-mining insects (Suppl. material 3).

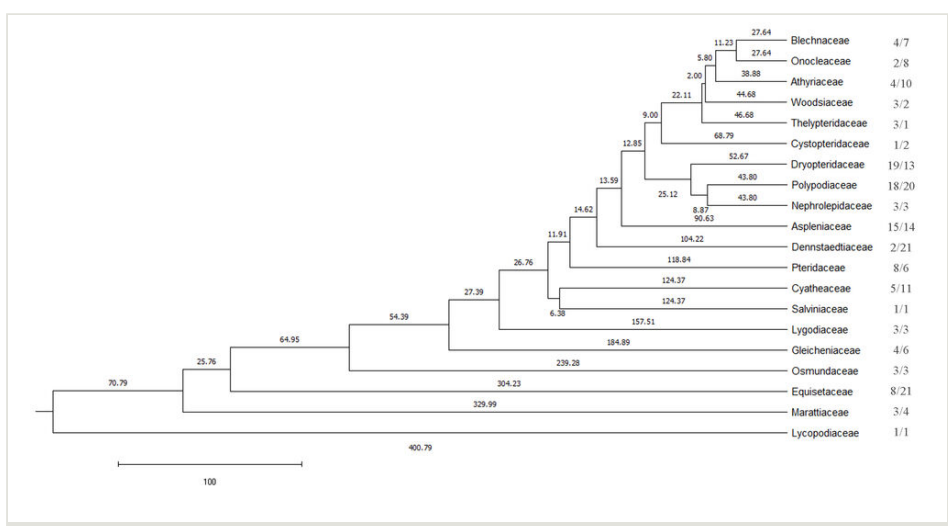

Figure 2. doi

The dated phylogenetic tree of host fern families generated with the $\mathrm{R}$ package ' $\mathrm{V}$. PhyloMaker' (Jin and Qian 2019). The first number after the fern family is the number of host fern species and the second is the number of fern miner species. The length of each branch is also shown and the scale bar unit is 100 myr. 
The number of host species was significantly and positively correlated with the total number of fern species at the family level $\left(R^{2}=0.614, t=5.352, P<0.001\right.$; Fig. 3a), but the number of miner species was not significantly correlated with the total number of fern species at the family level $\left(R^{2}=0.110, t=1.495, P=0.152\right.$; Fig. $\left.3 b\right)$.

$$
\text { a }
$$

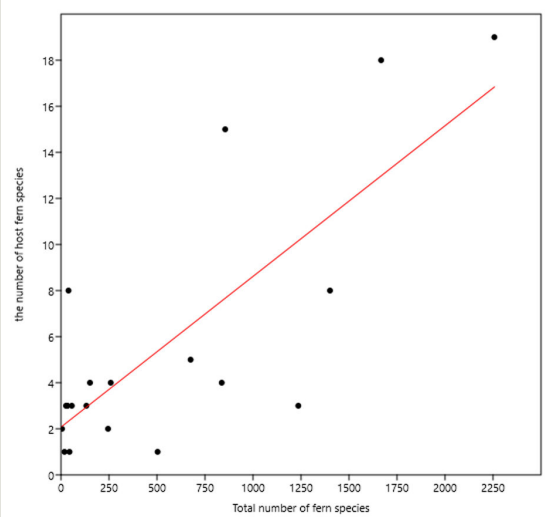

b

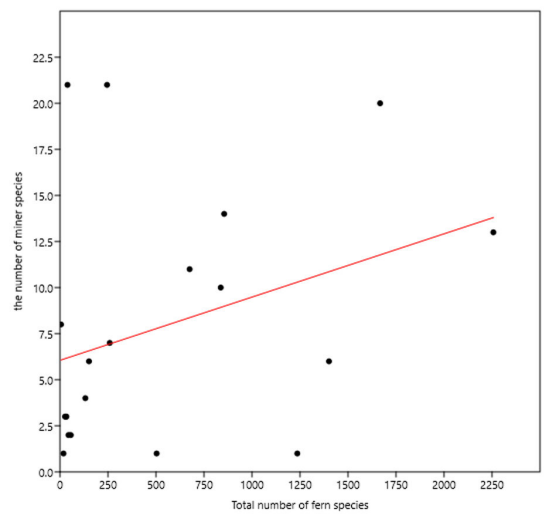

Figure 3.

Linear regressions between (a) the number of host species in each fern family; (b) the number of miner species for each fern family and the total number of fern species in the corresponding family. Families without any host species were not included.

\section{Discussion}

In this paper, we provide a preliminary checklist about fern miners and their host plants worldwide. Table 1 summarises this checklist in terms of published information to date. However, there is also more information available on some fern-mining groups and this is summarised here:

(1) Diptera: In Anthomyiidae, there is an unknown Chirosia species with Deparia acrostichoides as host in the Nearctic Region (Eiseman 2020b), while C. similata could be a possible Nearctic Pteridium borer (Eiseman 2018). In Drosophilidae, the Fuscoamoeba subgroup has many species that have been reared from rotting fern rachises (Magnacca et al. 2008). For Chromatomyia species in Agromyzidae, Kahanpää (2014) separates Chromatomyia and Napomyza as different genera (Kahanpää 2014) and Spencer (1990) considers that C. cheilanthus should belong to the genus Ptochomyza (Spencer 1990). Molecular phylogeny suggests that the genus of Phytomyza should include all species of Phytomyza, Chromatomyia, Napomyza and Ptochomyza (Winkler et al. 2009). However, only one fern-feeding Chromatomyia species is included in the above molecular analysis. Moreover, no Phytomyza s. s. species has previously been found as fern-mining. In this article, we rather kept the genus name of Chromatomyia and listed the Phytomyza species as the synonym of the corresponding Chromatomyia species in . 
(2) Lepidoptera: In Tineidae, early instar larvae of the subfamily Teichobinae are leaf miners, while their later instars feed on sporangia from a loose portable case (Gaedike 2019). An unknown species of Pyralidae has two hosts (Lygodium microphyllum and $L$. flexuosum) in the Oriental Region (Goolsby et al. 2003). There is an unknown moth in the Nearctic Region, which mines the leaves of Pteridium aquilinum (Eiseman 2020b), but the species name could not be confirmed. In Gelechiidae, Monochroa placidella larvae make gall-like deformities on the fronds of the bracken ( $P$. aquilinum) (Eiseman 2020b). Eiseman (personal observations) believes that the deformities are caused by internal feeding; he has also reared an undetermined Monochroa species from larvae that similarly bored in the terminal part of the rachis and caused a gall-like deformity.

(3) Hymenoptera: In Tenthredinidae, the genus Heptamelus has 36 species in the Palaearctic and Oriental Regions and their larvae are internal feeders and all probably use ferns as larva hosts (Vikberg and Liston 2009), but we cannot know with certainty which species of Heptamelus is involved, except for $H$. ochroleucus on Athyrium filix-femina (Vikberg 2017). With only 13 species and 3 tribes in Eurasia's temperate region, Blasticotomidae is a small family in the Hymenoptera and their larvae are stem borers on ferns (Taeger et al. 2010, Wikipedia 2019, Santiago-Blay 2004).

(4) Coleoptera: In Buprestidae, both Neotrachys and Endelus have fern-mining habits (Xiao 2018, Bellamy 1997). Most Neotrachys feed on the ferns of Cyatheaceae and Gleicheniaceae (Hespenheide 1980, Hespenheide 1982, Hespenheide 2006). However, some Neotrachys larvae may mine other non-fern plants. For example, $N$. dominicanus feeds on Arthrostylidium (Poaceae) (Meurgey 2017). The genus Leiopleura is morphologically similar and sometimes confused with Neotrachys, but Leiopleura feeds on Moraceae and Apocynaceae (Hespenheide 1991). Fern-feeding or not could be a clue to distinguish Neotrachys and its related genera. Although there are many publications on Endelus, only very few mention its host plants (Kalashian 2013).

Dominant plant groups generally are rich in leaf miners and rich in host plants, which could be explained by the 'plant apparency hypothesis' (Feeny 1976). Such phenomena have been found in several other leaf-mining insects (Dai et al. 2017, Dai et al. 2018). Apart from species richness in a fern taxonomic group, the distribution range should also be considered as an important component of 'plant apparency' (Dai et al. 2017). Equisetaceae has 39 species and eight host species, and Dennstaedtiaceae has 245 species and two host species, but both families host 21 miner species, which is the highest amongst all fern families (Fig. 2; Suppl. material 3). It is Equisetaceae and Dennstaedtiaceae that strongly affected the significance of the correlation in Fig. 3b. In particular, the bracken fern ( Pteridium aquilinum), one species in Dennstaetiaceae, had 20 miner species (Suppl. material 3), which is not less than many dominant flowering plants. The bracken fern might be the only globally distributed fern and one of the most widespread vascular plants, which occurs in temperate and subtropical regions in both hemispheres (Flora of North America Editorial Committee 1993). It is used as vegetable, food or feed in many places. It is also a common invasive plant in disturbed areas (Flora of North America Editorial Committee 1993). The above features of the bracken fern make it highly attractive to both miners and researchers, thus the high number of mining species might be the combined effects of 
plant apparency and sampling effects. Dryopteridaceae has 2257 species (Suppl. material 3 ) and also a cosmopolitan distribution, with many cultivated ornamental species (Olsen and Olsen 2007). Aspleniaceae has 855 species (Suppl. material 3) and also a worldwide distribution (POWO 2019). Polypodiaceae has 1667 species (Suppl. material 3) and is distributed nearly worldwide, but mainly in tropical areas, with some cultivated species (Simpson 2010). Both high species richness and wide geographical distribution could explain why the three families have large numbers of both host fern species and miner species. Besides $P$. aquilinum, Equisetum arvense (Equisetaceae), Athyrium filix-femina (Athyriaceae) and Matteuccia struthiopteris (Onocleaceae) also have a high richness of miners (10, 8 and 6 species, respectively) (Suppl. material 3). The common horsetail (E. arvense) is native throughout the Arctic and temperate regions in the Northern Hemisphere (Schaffner 1930). E. arvense becomes an invasive plant in New Zealand and a systematic evaluation of its potential biocontrol agents including miners and borers has been performed (Paynter and Barton 2008). The common lady-fern ( $A$. filix-femina) is one of the most abundant fern species in the temperate regions in the Northern Hemisphere (Adam 1995). The ostrich fern (M. struthiopteris) is widely distributed in the temperate regions of the Northern Hemisphere (Kimura et al. 2004). However, since the checklist of fern-mining insects and the corresponding host fern species is preliminary, these patterns need further verification.

As the sampling of fern miners and their hosts are insufficient in many places and some sampled records might be inaccessible, this study was only a preliminary list. We hope that this basic list can serve as an inital reference for future inventories and research on fernmining insects.

\section{Acknowledgements}

This work was supported by the National Natural Science Foundation of China (31760173 and 41971059) and the Science and Technology Project of Ganzhou City. Kevin Duffy acknowledges the support of the National Research Foundation of South Africa (Grant Numbers 131604). We also thank our colleague, Dr. Zhongyang Li for his significant assistance in our understanding why some fern families host many more miners. Thanks are also given to the two reviewers (Dr. Charles Eiseman and Dr. Yume Imada) for their valuable comments, which significantly improved the manuscript.

\section{References}

- $\quad$ Adam K (1995) Caffeic acid derivatives in fronds of the lady fern (Athyrium filix-femina). Phytochemistry 40 (5): 1577-1578. https://doi.org/10.1016/0031-9422(95)00536-g

- $\quad$ Alders CJM, Gielis C (1999) Apterona helicoidella, new for the Dutch fauna (Lepidoptera: Psychidae). Entomologische Berichten 59 (2): 21-22.

- Auerbach M, Hendrix S (1980) Insect-fern interactions: macrolepidopteran utilization and species-area association. Ecological Entomology 5 (2): 99-104. https://doi.org/ $\underline{\text { 10.1111/j.1365-2311.1980.tb01129.x }}$ 
- $\quad$ Barker M, Shaw S, Hickey RJ, Rawlins J, James W. F, (2005) Lepidopteran soral crypsis on Caribbean ferns. Biotropica 37 (2): 314-316. https://doi.org/10.1111/j. 1744-7429.2005.00040.x

- Bellamy CL (1997) Phylogenetic relationships of Xyroscelis (Coleoptera: Buprestidae. Invertebrate Systematics 11 (4): 569-574.

- Beneš K (2014) Sawflies (Hymenoptera, Symphyta) of the Bohemian Forest and its foothills. Silva Gabreta 20 (3): 131-147.

- Biological Records Centre (2020) United Kingdom Beetle Recording. URL: https:// www.coleoptera.org.uk

- $\quad$ Bird H (2012) The fern-feeding species of Papaipema ; No. 25 (Lepidoptera). The Canadian Entomologist 59 (4): 92-96. https://doi.org/10.4039/Ent5992-4

- Brown VK, McGavin GC (2007) The biology of some mine and gall forming Diptera on bracken, Pteridium aquilinum (L.) Kühn. Journal of Natural History 16 (4): 511-518. https://doi.org/10.1080/00222938200770411

- $\quad$ Center TD, Dray FA, Jubinsky GP, Grodowitz MJ (2002) Insects and other arthropods that feed on aquatic and wetland plants. United States Department of Agriculture, Agricultural Research Service

- Chaloux, Andrea (2012) Online conservation guide for Papaipema stenocelis. URL: https://guides.nynhp.org/chain-fern-borer-moth

- Chandra S, Srivastava M (2003) Pteridology in the new millennium. Springer Netherlands, Dordrecht. [ISBN 978-94-017-2811-9] https://doi.org/ 10.1007/978-94-017-2811-9

- $\quad$ Civelek HS (2002) New records of Agromyzidae (Diptera) from Western Turkey. Insecta Mundi 16 (1-3): 49-55. URL: http://digitalcommons.unl.edu/insectamundi/538

- Correia P, Bashforth A, Šimůnek Z, Cleal C, Sá A, Labandeira C (2020) The history of herbivory on Sphenophytes: A new Calamitalean with an insect hall from the Upper Pennsylvanian of Portugal and a review of arthropod herbivory on an ancient lineage. International Journal of Plant Sciences 181 (4): 387-418. https://doi.org/10.1086/707105

- $\quad$ Crang R, Lyons-Sobaski S, Wise R (2018) Plant anatomy: a concept-based approach to the structure of seed plants. Springer

- Dai X, Zhang W, Xu J, Duffy KJ, Guo Q (2017) Global pattern of plant utilization across different organisms: Does plant apparency or plant phylogeny matter? Ecology and Evolution 7 (8): 2535-2545. https://doi.org/10.1002/ece3.2882

- Dai X, Long C, Xu J, Guo Q, Zhang W, Zhang Z, Bater (2018) Are dominant plant species more susceptible to leaf-mining insects? A case study at Saihanwula Nature Reserve, China. Ecology and Evolution 8 (15): 7633-7648. https://doi.org/10.1002/ ece3.4284

- Dai X, Chen C, Li Z, Wang X (2020) Taxonomic, phylogenetic, and functional diversity of ferns at three differently disturbed sitesin Longnan County, China. Diversity 12 (4): 135. https://doi.org/10.3390/d12040135

- $\quad$ Dempewolf M (2001) Larvalmorphologie und Phylogenie der Agromyzidae (Diptera). Philosophiae Doctor Thesis, University of Bielefeld, Bielefeld, Germany.

- De Prins J, De Prins W (2020) Global taxonomic database of Gracillariidae (Lepidoptera). URL: http://www.gracillariidae.net/

- $\quad$ Edmunds R, Kimber I, Talbot P, Manning D, Dickerson B, Plant C, Ellis M (2020) British leafminers. URL: http://www.leafmines.co.uk 
- Eiseman C (2018) New rearing records for muscoid leafminers (Diptera: Anthomyiidae, Scathophagidae) in the United States. Proceedings of the Entomological Society of Washington 120 (1): 25-50. https://doi.org/10.4289/0013-8797.120.1.25

- $\quad$ Eiseman C (2020a) Further Nearctic rearing records for phytophagous muscoid flies (Diptera: Anthomyiidae, Scathophagidae). Proceedings of the Entomological Society of Washington 122 (3): 595-603. https://doi.org/10.4289/0013-8797.122.3.595

- Eiseman C (2020b) Leafminers of North America. Second Edition. Charley Eiseman URL: http://charleyeiseman.com/leafminers/

- Ellis W (2020) Plant parasites of Europe leafminers, galls and fungi. URL: https:// bladmineerders.nl/

- Feeny P (1976) Plant apparency and chemical defense. Recent Advances in Phytochemistry 10: 1-40.

- $\quad$ Flora of North America Editorial Committee (1993) Flora of North America: Volume 2: Pteridophytes and Gymnosperms. Oxford University Press, New York. URL: https:// books.google.com/books?id=VnIBQMJOi-UC [ISBN 9780195082425]

- Fountain-Jones N, McQuillan P, Grove S (2012) Beetle communities associated with the tree fern Dicksonia antarctica Labill. in Tasmania. Australian Journal of Entomology 51 (3): 154-165. https://doi.org/10.1111/j.1440-6055.2011.00855.x

- Gaedike R (2019) Tineidae II (Myrmecozelinae, Perissomasticinae, Tineinae, Hieroxestinae, Teichobiinae and Stathmopolitinae). Brill [ISBN 9789004387515] https:// doi.org/10.1163/9789004387515

- George P (2014) Insect herbivores of horsetails: bionomics, dispersal, and co-evolution. American Entomologist 60 (4): 235-240. https://doi.org/10.1093/ae/60.4.235

- $\quad$ Gerson U (1979) The associations between pterydophytes and arthropods. Veterinary Clinics of North America 5 (2): 177-195.

- Goolsby J, Wright A, Pemberton R, et al. (2003) Exploratory surveys in Australia and Asia for natural enemies of Old World climbing fern, Lygodium microphyllum: Lygodiaceae. Biological Control 28 (1): 33-46. https://doi.org/10.1016/ S1049-9644(03)00054-9

- $\quad$ Gosik R (2009) Description of the mature larva and pupa of Bagous lutulentus (Gyllenhal), with comments on its biology (Coleoptera: Curculionidae).

Gastroentérologie Clinique Et Biologique 34 (10): 534-540.

- Gosik R, Wanat M (2014) First descriptions of immature stages of the weevils Bagous elegans, B. aliciae, and B. Iutulosus. Journal of Insect Science 14 (141): 1-13. https:// doi.org/10.1093/jisesa/ieu003

- $\quad$ Gosik R, Mazur MA, Sawka-Gadek N (2019) Firstdescriptions of larva and pupa of Bagous claudicans Boheman, 1845 (Curculionidae, Bagoinae) and systematic position of the species based on molecular and morphological data. Insects 10 (6): 166. https:// doi.org/10.3390/insects10060166

- $\quad$ Grehan JR (1989) Larval feeding habits of the Hepialidae (Lepidoptera). Journal of Natural History 23 (4): 803-824. https://doi.org/10.1080/00222938900770421

- Hammer $\varnothing$, Harper DA, Ryan PD (2001) PAST: paleontological statistics software package for education and data analysis. Palaeontologia Electronica 4 (1): 1-9.

- Hawkeswood TJ (2003) Observations on the biology and host plants of the Australian flea beetle, Halticorcus platycerii Lea, 1917 (Coleoptera: Chrysomelidae: Alticinae). Calodema 1: 31-34. 
- Heckford RJ (2004) A consideration of the latvae of Psyckoides verhuella Btuand, 1853, and P. filicivora (Meyrick, 1937) (Lepidoptera: Tineidae). Entomologists Gazette 55 (4): 211-214.

- Hering EM (1951) Biology of the leaf miners. Springer, Dordrecht, Berlin. https://doi.org/ 10.1007/978-94-015-7196-8

- Hespenheide H (1980) A Revision of Antillean Neotrachys (Coleoptera, Buprestidae). Journal of the Kansas Entomological Society 53 (4): 815-824. URL: http://www.jstor.org/ stable/25084115

- Hespenheide H (1982) A revision of Central American species of Neotrachys (Coleoptera, Buprestidae). The Coleopterists Bulletin 36 (2): 328-349.

- Hespenheide H (2006) New species and new records of Neotrachys Obenberger, 1923, from Central America and the Antilles (Coleoptera: Buprestidae). The Pan-pacific Entomologist 82 (2): 223-241.

- Hespenheide HA (1991) Bionomics of Leaf-Mining Insects. Annual Review of Entomology 36 (1): 535-560. https://doi.org/10.1146/annurev.en.36.010191.002535

- Hill MP, Cilliers CJ (1999) Azolla filiculoides Lamarck (Pteridophyta: Azollaceae), its status in South Africa and control. Hydrobiologia 415: 203-206. https://doi.org/10.1023/ A:1003869230591

- Hinz L, Zahniser J (2015) Review andupdate of non-mollusk invertebrate species in greatest need of conservation: final report. Illinois Natural History Survey

- Isowa R, Kojima H (2011) Larval morphology and biology of Halticorcus kasuga (Nakane) (Coleoptera, Chrysomelidae). Elytra, Tokyo, New Series 1 (2): 327-333.

- Jin Y, Qian H (2019) V.PhyloMaker: an R package that can generate very large phylogenies for vascular plants. Ecography 42 (8): 1353-1359. https://doi.org/10.1111/ ecog. 04434

- Jolivet P (1991) Sélection trophique chez les Alticinae (Coleoptera Chrysomelidae) (suite). Bulletin mensuel de la Société linnéenne de Lyon 60 (2): 53-72. https://doi.org/ 10.3406/linly.1991.10922

- Kahanpää J (2014) Checklist of the leaf-mining flies (Diptera, Agromyzidae) of Finland. ZooKeys 441: 291-303. https://doi.org/10.3897/zookeys.441.7586

- Kalashian M (2013) Two new species of the genus Endelus Deyrolle, 1864 (Coleoptera: Buprestidae) from Malaysia and the Philippines with notes on the synonymy of some species of the subgenus Endelus s. str. Кавказский энтомол. бюллетень 9 (1): 83-88.

- Kato M (1991) Leaf-mining chrysomelids reared from pteridophytes. Japanese Journal of Entomology 59: 671-674.

- Kato M (2002) First record of herbivory on Lycopodiaceae (Lycopodiales) by a dipteran (Pallopteridae) leaf/stem-miner. Canadian Entomologist 134 (5): 699-701. https:// doi.org/10.4039/Ent134699-5

- $\quad$ Kawahara AY, Tangalin NB, Rubinoff D (2011) Life-history notes on the fern-mining endemic Hyposmocoma (Euperissus) trivitella Swezey 1913 (Lepidoptera:

Cosmopterigidae) from Kauai and a report of associated parasitoids (Hymenoptera: Bethylidae). Proceedings of the Hawaiian Entomological Society 43: 9-12. URL: http:// hdl.handle.net/10125/21672

- Kim S, Bae YS (2007) A new species of Psychoides Bruand (Lepidoptera, Tineidae, Teichobiinae) from Korea, with some biological information. Journal of Asia-Pacific Entomology 10 (1): 21-26. https://doi.org/10.1016/S1226-8615(08)60326-X 
- $\quad$ Kimura T, Suzuki M, Takenaka M, Yamagishi K, Shinmoto H (2004) L-Ocaffeoylhomoserine from Matteuccia struthiopteris. Phytochemistry 65 (4): 423-426. https://doi.org/10.1016/i.phytochem.2003.10.008

- Konstantinov AS, Prathapan KD (2008) New generic synonyms in the oriental flea beetles (Coleoptera: Chrysomelidae). The Coleopterists Bulletin 62 (3): 381-418. https:// doi.org/10.1649/1089.1

- Kwon YJ, Suh SJ (1982) Classification of the Anthomyiidae from Korea. II. (Diptera : Calyptratae). Korean Journal of Applied Entomology 21 (4): 179-184.

- Lafontaine D, Schmidt C (2010) Annotated check list of the Noctuoidea (Insecta, Lepidoptera) of North America north of Mexico. ZooKeys 40: 1-239. https://doi.org/ 10.3897/zookeys.40.414

- Lawton JH (1976) The structure of the arthropod community on bracken. Botanical Journal of the Linnean Society 73 (1-3): 187-216. https://doi.org/10.1111/j. 1095-8339.1976.tb02022.x

- Lawton JH (1982) Vacantniches and unsaturated communities: a comparison of bracken herbivores at sites on two continents. Journal of Animal Ecology 51 (2): 573-595.

- $\quad$ Liston A (2007) Zur Biologie und Vorkommen von Blasticotoma filiceti Klug, 1834 (Hymenoptera, Blasticotomidae) in Brandenburg und Berlin. Entomologische Nachrichten und Berichte 51: 95-99.

- Liu W, Dai X, Xu J (2015) Influences of leaf-mining insects on their host plants: A review. Collectanea Botánica 34: e005.

- Lonsdale O (2017) The Liriomyza (Diptera: Schizophora: Agromyzidae) of Canada \& Alaska. Zootaxa 4234: 1-156. https://doi.org/10.11646/zootaxa.4234.1.1

- MacGarvin M, Lawton J, Heads P (1986) The herbivorous insect communities of open and woodland bracken: observations, experiments and habitat manipulations. Oikos 47 (2): 135-148. https://doi.org/10.2307/3566038

- Magnacca K, Foote D, O'Grady P (2008) A review of the endemic Hawaiian Drosophilidae and their host plants. Zootaxa 1728: 1-58. https://doi.org/10.11646/ zootaxa.1728.1.1

- Magnacca KN, O'Grady PM (2014) Revision of the modified mouthparts species group of Hawaiian Drosophila (Diptera: Drosophilidae): The Ceratostoma, Freycinetiae, Semifuscata, and Setiger subgroups, and unplaced species. 130 . University of California Press

- Maunsell SC, Burwell CJ, Morris RJ, Mcdonald WJ, Edwards ED, Oberprieler RG, Kitching RL (2016) Host-plants of leaf-miners in Australian subtropical rainforest. Austral Entomology 56 (4): 403-411. https://doi.org/10.1111/aen.12252

- Mcgavin G, Brown V (1986) Variation in populations of mine- and gall-forming Diptera and the growth form of their host plant, bracken (Pteridium aquilinum (L.) Kuhn). Annals \& Magazine of Natural History 20 (4): 799-816.

- Mehltreter K, Walker L, Sharpe J (2010) Fern Ecology. Cambridge University Press, New York. https://doi.org/10.1017/CBO9780511844898

- Meurgey F (2017) Observations sur une plante hôte de Neotrachys dominicanus Théry, 1947 en Guadeloupe (Coleoptera, Buprestidae). Contribution à l'étude des Coléoptères des Petites Antilles 3: 53-54.

- $\quad$ Michelsen V, Palmer MW (2020) Pegomya disticha Griffiths and P. cedrica Huckett (Diptera: Anthomyiidae)—first documented case of insects trespassing the silica barrier 
of Common scouring-rush, Equisetum hyemale L. Zootaxa 4718 (3): 4. https://doi.org/ 10.11646/zootaxa.4718.3.4

- Morrone J (2002) Biogeographical regions under track and cladistic scrutiny. Journal of Biogeography 29 (2): 149-152. https://doi.org/10.1046/j.1365-2699.2002.00662.x

- Muus TST (2015) De opmerkelijke ontdekking van Psychoides verhuella (Lepidoptera: Tineidae), nieuw in Nederland. Entomologische Berichten 75 (1): 11-14.

- $\quad$ Nadein K (2013) Febraina: a new subtribe of Alticini with cladistic analysis based on morphology (Coleoptera: Chrysomelidae: Galerucinae). Systematic Entomology 38 (3): 491-506. https://doi.org/10.1111/syen.12007

- Novgorodova TA, Biryukova O (2011) Some ethological aspects of the trophobiotic interrelations between ants (Hymenoptera: Formicidae) and larvae of the sawfly Blasticotoma filiceti (Hymenoptera: Blasticotomidae). European Journal of Entomology 108 (1): 47-52. https://doi.org/10.14411/eje.2011.006

- $\quad$ Olsen S, Olsen S (2007) Encyclopedia of garden ferns. Timber Press

- $\quad$ Oppenheim SJ, Feindt W, DeSalle R, Goldstein PZ (2018) De Novo characterization of transcriptomes from two North American Papaipema stem-borers (Lepidoptera: Noctuidae). PLOS One 13 (1): e0191061. https://doi.org/10.1371/journal.pone.0191061

- Patra B, Bera S (2007) Herbivore damage to ferns caused by a chrysomelid beetle from Lower Gangetic Plains of West Bengal, India. American Fern Journal 97 (1): 19-29. https://doi.org/10.1640/0002-8444(2007)97[19:HDTFCB]2.0.CO;2

- Patra B, Bera S, Hickey RJ (2008) Soral crypsis: protective mimicry of a coccid on an Indian fern. Journal of Integrative Plant Biology 50 (6): 653-658. https://doi.org/10.1111/j. 1744-7909.2008.00664.x

- $\quad$ Patrick B (2015) Leather-leaf fern's moth fauna. The Weta 49: 23-27.

- Paynter Q, Barton J (2008) Prospects for biological control of field horsetail Equisetum arvense L. in New Zealand. Landcare Research Contract Report LC0708/100. Landcare Research, Auckland, New Zealand.

- $\quad$ Pitkin B, Ellis W, Plant C, Edmunds R (2019) The leaf and stem mines of British flies and other insect. URL: http://www.ukflymines.co.uk

- Poinar G (2014) Insect herbivores of horsetails: Bionomics, dispersal, and co-evolution. American Entomologist 60 (4): 235-240. https://doi.org/10.1093/ae/60.4.235

- POWO (2019) Plants of the world online. Facilitated by the Royal Botanic Gardens, Kewscience. http://www. plantsoftheworldonline.org. Accessed on: 2020-12-21.

- $\quad$ Richerson PJ, Grigarick AA (1967) The Life History of Stenopelmus rufinasus (Coleoptera: Curculionidae). Annals of the Entomological Society of America 60 (2): 351-354. https://doi.org/10.1093/aesa/60.2.351

- $\quad$ Rigby C, Lawton J (1981) Species-area relationships of arthropods on host plants: herbivores on bracken. Journal of Biogeography 8 (2): 125-133. https://doi.org/ 10.2307/2844555

- Samuelson GA (1973) Alticinae of Oceania (Coleoptera: Chrysomelidae). Pacific insects monograph 30: 1-165.

- Santiago-Blay J (2004) Leaf-mining chrysomelids. In: Jolivet P, Santiago-Blay JA, Schmitt M (Eds) New Developments on the Biology of Chrysomelidae. SPB Academic Publishing, The Hague, The Netherlands, $81 \mathrm{pp}$.

- Sasakawa M (2010) Notes on the Japanese fern-miners (Diptera, Agromyzidae), with description of a new species. Japanese Journal of Systematic Entomology 16 (1): 161-164. 
- Schaffner J (1930) Geographic distribution of the species of Equisetum in relation to their phylogeny. American Fern Journal 20 (3): 89-106. https://doi.org/10.2307/1543867

- Schneider H, Schuettpelz E, Pryer KM, Cranfill R, Magallon S, Lupia R (2004) Ferns diversified in the shadow of angiosperms. Nature 428 (6982): 553-557. https://doi.org/ 10.1038/nature02361

- Schwarz M (2005) Die Selandriinae außer der Gattung Dolerus (Hymenoptera, Symphyta, Tenthredinidae) Oberösterreichs (Österreich). Beiträge zur Naturkunde Oberösterreichs 14: 435-450.

- $\quad$ Schweitzer DF (2012) Papaipema sp. 2 nr. pterisiiOstrich Fern Borer Moth. https:// explorer.natureserve.org/Taxon/ELEMENT GLOBAL.2.111682/

\section{Papaipema sp 2 nr_pterisii}

- $\quad$ Shcherbakov DE (2006) Fern sawfly larvae Blasticotoma filiceti Klug, 1834

(Hymenoptera: Blasticotomidae) are visited by ants: a new kind of trophobiosis. Russian Entomological Journal 15 (1): 67-72.

- Shcherbakov DE (2008) New records of Hymenoptera from the Moscow Region and other parts of Russia, with notes on synonymy of Konowia species. Russian Entomological Journal 17 (2): 209-212.

- $\quad$ Shiao S, Wu S (2005) A new fern-feeding species of Agromyzidae (Diptera) from Taiwan. Transactions of the American Entomological Society 131 (3-4): 355-363.

- $\quad$ Simpson M (2010) Plant Systematics. Second Edition. Academic Press, 752 pp.

- $\quad$ Sinclair R, Hughes $L$ (2008) Incidence of leaf mining in different vegetation types across rainfall, canopy cover and latitudinal gradients. Austral Ecology 33 (3): 353-360. https:// doi.org/10.1111/j.1442-9993.2007.01825.x

- $\quad$ Sinclair RJ, Hughes L (2008) Leaf mining in the Myrtaceae. Ecological Entomology 33 (5): 623-630. https://doi.org/10.1111/j.1365-2311.2008.01014.x

- $\quad$ Sinclair RJ, Hughes L (2010) Leaf miners: The hidden herbivores. Austral Ecology 35 (3): 300-313. https://doi.org/10.1111/j.1442-9993.2009.02039.x

- Smith S, Brown J (2018) Constructing a broadly inclusive seed plant phylogeny. American Journal of Botany 105 (3): 302-314. https://doi.org/10.1002/ajb2.1019

- Solis MA, Davis DR, Nishida K (2005a) Life history and systematics of Albusambia elaphoglossumae (Lepidoptera: Crambidae): a new genus and species of musotimine with leaf-mining biology from Costa Rica. Revista de Biología Tropical 53 (3-4):

487-501. https://doi.org/10.15517/rbt.v53i3-4.14617

- Solis MA, Yen S, Goolsby J, Wright T, Pemberton R, Winotai A, Chattrukul U, Thagong A, Rimbut S (2005b) Siamusotima aranea, anew stem-boring Musotimine (Lepidoptera: Crambidae) from Thailand feeding on Lygodium flexuosum (Schizaeaceae). Annals of the Entomological Society of America 98 (6): 887-895. https://doi.org/

10.1603/0013-8746(2005)098[0887:Saansm]2.0.Co;2

- Solis MA, Pratt P, Makinson J, Zonneveld R, Lake E (2017) Another new Lygodiumboring species of the Musotimine genus Siamusotima (Lepidoptera: Crambidae) from China. Proceedings of the Entomological Society of Washington 119 (3): 471-480. https://doi.org/10.4289/0013-8797.119.3.471

- Spencer KA (1976) The Agromyzidae of New Zealand (Insecta: Diptera). Journal of the Royal Society of New Zealand 6 (2): 153-211. https://doi.org/ 10.1080/03036758.1976.10421470

- $\quad$ Spencer KA (1990) Host specialization in the World Agromyzidae (Diptera). Springer Netherlands https://doi.org/10.1007/978-94-009-1874-0 
- Suwa M (1984) Supplementary notes on the family Anthomyiidae of Japan (Diptera), VII. Insecta Matsumurana 10: 87-106.

- Suwa M (1999) Japanese records of Anthomyiidae files (Diptera: Anthomyiidae). Insecta Matsumurana 55: 203-244. URL: http://hdl.handle.net/2115/9895

- $\quad$ Suwa M (2005) Supplementary notes on the family Anthomyiidae of Japan (Diptera), 6. Insecta matsumurana. New series : journal of the Faculty of Agriculture Hokkaido University, series entomology. 61: 87-106. URL: http://hdl.handle.net/2115/9924

- Suwa M (2013) Anthomyiid flies from Sakhalin (Diptera: Anthomyiidae). Insecta Matsumurana 69 (1): 133-194.

- $\quad$ Taeger A, Blank S, Liston A (2010) World catalog of Symphyta (Hymenoptera). 2580. Zootaxa, 1063 pp. https://doi.org/10.11646/zootaxa.2580.1.1

- Vikberg V, Liston A (2009) Taxonomy and biology of European Heptamelini (Hymenoptera, Tenthredinidae, Selandriinae). Zootaxa 2112 (2112): 1-24. https:// doi.org/10.11646/zootaxa.2112.1.1

- Vikberg V (2017) Two sawflies new for the fauna of Russia, one being new for Europe and the western Palaearctic region (Hymenoptera: Symphyta: Heptamelidae and Tenthredinidae). Sahlbergia 23 (1): 17-19.

- Weintraub J, Lawton J, Scoble M (1995) Lithinine moths on ferns: a phylogenetic study of insect-plant interactions. Biological Journal of the Linnean Society 55 (3): 239-250. https://doi.org/10.1111/j.1095-8312.1995.tb01062.x

- Wikipedia (2019) Blasticotomidae. URL: https://en.wikipedia.org/wiki/Blasticotomidae

- Winkler IS, Scheffer SJ, Mitter C (2009) Molecular phylogeny and systematics of leafmining flies (Diptera: Agromyzidae): delimitation of Phytomyza Fallén sensu lato and included species groups, with new insights on morphological and host-use evolution. Systematic Entomology 34: 260-292. https://doi.org/10.1111/j.1365-3113.2008.00462.x

- Woodley NE, Janzen DH (1995) A new species of Melanagromyza (Diptera: Agromyzidae) mining leaves of Bromelia pinguin (Bromeliaceae) in a dry forest in Costa Rica. Journal of Natural History 29 (5): 1329-1337.

- Xiao L (2018) Morphological Taxonomy and Molecular Phylogeny of Common Leafmining Jewel Beetles in Jiangxi Province. Jiangxi Agricultural University, 69 pp. URL: https://kns.cnki.net/kcms/detail/detail.aspx?

FileName=1018275706.nh\&DbName=CMFD2019

- Zanne A, Tank D, Cornwell W, Eastman J, Smith S, FitzJohn R, McGlinn D, O'Meara B, Moles A, Reich P, Royer D, Soltis D, Stevens P, Westoby M, Wright I, Aarssen L, Bertin R, Calaminus A, Govaerts R, Hemmings F, Leishman M, Oleksyn J, Soltis P, Swenson $\mathrm{N}$, Warman L, Beaulieu J (2014) Three keys to the radiation of angiosperms into freezing environments. Nature 506 (7486): 89-92. https://doi.org/10.1038/nature12872

\section{Supplementary materials}

\section{Suppl. material 1: The feeding mode and feeding plant organ of each fern miner doi}

Authors: Jie Yang, Xiaohua Dai

Data type: Feeding habits

Download file $(16.21 \mathrm{~kb})$ 
Suppl. material 2: The number of fern-miners in each insect family and each insect order doi

Authors: Jie Yang, Xiaohua Dai

Data type: Number of species

Download file (16.04 kb)

Suppl. material 3: The number of miners on each fern species, each fern family and each fern order doi

Authors: Jie Yang, Xiaohua Dai

Data type: Number of species

Download file $(20.77 \mathrm{~kb})$ 